\title{
LINEAR MEROMORPHIC DIFFERENTIAL EQUATIONS: A MODERN POINT OF VIEW
}

\author{
V. S. VARADARAJAN
}

\begin{abstract}
A large part of the modern theory of differential equations in the complex domain is concerned with regular singularities and holonomic systems. However the theory of differential equations with irregular singularities has a long history and has become very active in recent years. Substantial links of this theory to the theory of algebraic groups, commutative algebra, resurgent functions, and Galois differential methods have been discovered. This survey attempts a general introduction to some of these aspects, with emphasis on reduction theory, asymptotic analysis, Stokes phenomena, and certain moduli problems.
\end{abstract}

\section{INTRODUCTION}

I would like to give a brief outline of some of the advances made in recent years in the theory of ordinary linear differential equations in the complex domain with meromorphic coefficients. This is a vast area with connections to many parts of contemporary mathematics such as commutative algebra, algebraic geometry, algebraic groups, complex analysis (both conventional and resurgent), and so on. To give a comprehensive survey of this subject is thus impossible and I will not attempt it here. I shall only restrict myself to a few aspects with which I have some familiarity and which have been discussed in detail in my papers with D. G. Babbitt $[8(\mathrm{a})-(\mathrm{g})]$. For treatments of topics not discussed here, as well as additional perspective and more complete references, I refer the reader to V. Arnold's encyclopaedia article [3], my own paper in the Expositiones Mathematicae [4(a)], the Bourbaki talks of D. Bertrand [5(a)(b)] and A. Beauville [5(c)], the recent books of D. V. Anosov and A. Bolibruch [6(a)], of Iwasaki et al. [6(c)], the papers of Iwasaki $[6(\mathrm{~d})(\mathrm{e})]$, of M. F. Singer $[6(\mathrm{f})(\mathrm{g})]$, and the many papers of J. P. Ramis [7] (which I shall refer to more precisely later) that link the theory of ordinary differential equations with (Gevrey type of) asymptotic analysis and the theory of resurgent functions in a major way. Finally, as a general philosophical guide to the themes discussed here I refer the reader to the celebrated and highly influential monograph $[9(\mathrm{a})]$ of P. Deligne. However, to give the reader a chance to understand the many threads of thought that go through the subject, I shall begin with a brief survey of the classical themes and ideas. In the next section I shall give a brief discussion of the framework for the modern view that grows out of the classical sources,

Received by the editors October 24, 1994, and, in revised form, June 22, 1995.

1991 Mathematics Subject Classification. Primary 34A20, 13N05.

This is a revised and expanded version of an invited hour talk at the AMS meeting in Portland, Oregon, June 15, 1991. Due to various personal circumstances its preparation has been delayed till now. 
encompasses it, and allows one not only to formulate the classical theories in very general settings but to generalize them in a far-reaching manner. It has led to a lot of progress and understanding of the old problems and suggested new problems as well. I believe that it is still very worthwhile to go back to the classical theories and attempt to study them with the help of the ideas and techniques characteristic of the more recent developments.

Riemann: monodromy of the hypergeometric functions. The origins of the subject may be traced to the famous paper of Riemann in 1857 on the hypergeometric functions in which, among other things, he calculated the global monodromy of these functions [1]. As long as a differential equation (of the $n^{\text {th }}$ order or a system of first order in $\mathbf{C}^{n}$ ) is viewed in the neighborhood of a point, say $z_{0}$, where all the coefficients of the equation are regular, nothing of interest happens beyond the fact that any initial data at $z_{0}$ determines the solution uniquely around $z_{0}$ as a holomorphic function; and the linear character of the differential equation guarantees that this solution may be analytically continued along any path that does not encounter any singularity of the coefficients. Moreover the result of the analytic continuation, which depends on the path of course, does not change if the path is deformed continuously. Consequently, by considering loops starting and ending at $z_{0}$, one obtains an action of the homotopy group of loops on the $n$-dimensional space $V$ of germs of local solutions at $z_{0}$; the loops have to be restricted to be in the domain of regularity of the coefficients. The image of this action in $G L(V)$ is classically known as the monodromy group of the equation.

It was Riemann who introduced the monodromy group, understood its significance for the problem of the global description of the solutions of the differential equation in question, and computed it explicitly for the hypergeometric differential equation

$$
z(z-1) \frac{d^{2} F}{d z^{2}}+[(a+b+1) z-c] \frac{d F}{d z}+a b F=0 .
$$

The hypergeometric series

$$
F(a, b, c ; z)=1+\frac{a b}{1 . c} z+\frac{a(a+1) b(b+1)}{1.2 c(c+1)} z^{2}+\ldots \quad(|z|<1)
$$

is a solution and the issue is its analytic continuation. The singularities of the coefficients, which are determined by dividing by $z(z-1)$ so as to make the leading coefficient 1 , are the points $0,1, \infty$ in $\mathbf{P}_{1}$. The fundamental group of $\mathbf{P}_{1} \backslash\{0,1, \infty\}$ is the free group on two generators $\lambda_{0}, \lambda_{1}$, the loops around 0,1 respectively. The classical method of indicial equations determines the exponents at the singularities; Riemann determined the $2 \times 2$ matrices $M_{0}, M_{1}$ representing the loops $\lambda_{0}, \lambda_{1}$ in terms of the exponents.

Riemann restricted himself only to equations whose solutions around the singular points belong to the module generated by the multivalued functions $z^{a}(a \in \mathbf{C})$; subsequently the functions $z^{a}(\log z)^{m}(m \in \mathbf{Z}, \geq 0)$ were also allowed. This is equivalent to supposing that the solutions have only polynomial growth at the singular point if we assume it to be $\infty$. The work of Riemann and his successors Fuchs, Schwarz, Klein, Poincaré, Pochhammer and others (see the fascinating accounts of Gray [2]) showed that the theory of the equations with singularities only of the type described above, the so-called regular singularities, was a beautiful structure, many 
aspects of which were worth exploring intensely, such as the "number of parameters" on which the equations depend when one fixes the singularities, the connection between the exponents and the monodromy group for equations with higher order and/or larger number of singularities than the hypergeometric equation, the study of what happens under "confluence", i.e., the phenomenon when two singularities are allowed to coalesce, the determination of when the monodromy group is finite or discrete, and so on.

Hilbert: the Riemann-Hilbert problem. In 1900 Hilbert lifted the subject to an entirely new level when he posed the penetrating problem of showing that it was always possible to determine a differential equation of the Fuchsian class with given singularities and monodromy group. One may interpret the question in many ways as it is somewhat vague; one way is as follows: given finite set $F$ of singularities in $\mathbf{P}_{1}$ and a given representation of the fundamental group of $\mathbf{P}_{1} \backslash F$, the problem is to construct a differential equation with rational coefficients and regular singularities, these being precisely the points of $F$, whose monodromy representation is the given one. This (in many versions) is the so-called RiemannHilbert problem; it was immediately solved for the case of dimension 2 by Hilbert and Schlesinger. Its general solution (with some caveats which were not fully understood till very much later, indeed only in more recent times) came sometime later, and the effort to understand its many ramifications has had a tremendous impact on the development of the subject. The themes generated by the Riemann-Hilbert problem show no signs of dying out. Especially fertile has been the circle of ideas related to the completely integrable system of partial differential equations discovered by Schlesinger that describe the variation of the coefficients and the singularities when one imposes the restriction that the monodromy does not change.

Appell, Picard: hypergeometric functions of several variables. One must also mention here the generalizations to several variables of the hypergeometric functions that were discovered by Appell and Picard at the turn of this century. Unlike the situation for ordinary differential equations, we now have partial differential equations and one has to impose the condition of integrability that will guarantee that any initial data determines a local solution. For example, write $(x, k):=x(x+1) \ldots(x+k-1)$ and let

$$
F\left(a_{1}, \ldots, a_{d}, b, c, z_{1}, \ldots, z_{d}\right)
$$

be the series

$$
\sum_{m_{1}, \ldots, m_{d} \geq 0} \frac{\left(a_{1}, m_{1}\right) \ldots\left(a_{d}, m_{d}\right)\left(b, m_{1}+\cdots+m_{d}\right)}{\left(c, m_{1}+\cdots+m_{d}\right)\left(1, m_{1}\right) \ldots\left(1, m_{d}\right)} z_{1}^{m_{1}} \ldots z_{d}^{m_{d}} .
$$

Let $\theta_{j}=z_{j} \partial / \partial z_{j}$ and let

$$
Y=\left(\begin{array}{c}
F \\
\theta_{1} F \\
\vdots \\
\theta_{d} F
\end{array}\right)
$$

Then we have the system of differential equations

$$
\frac{\partial}{\partial z_{j}} Y=A_{j} Y \quad(1 \leq j \leq d)
$$


where $\Gamma:=\sum_{j} A_{j} d z_{j}$ is of the form

$$
\Gamma=\sum_{j} C_{j, 0} \frac{d z_{j}}{z_{j}}+\sum_{j} C_{j, 1} \frac{d z_{j}}{z_{j}-1}+\sum_{i<j} C_{i, j} \frac{d\left(z_{i}-z_{j}\right)}{z_{i}-z_{j}},
$$

the $C$ 's being constant $(d+1) \times(d+1)$ matrices. This is an integrable system defined on $\left(\mathbf{P}_{1}\right)^{d} \backslash F$ where $F$ is the union of the hyperplanes given by the equations

$$
z_{j}=0,1, \infty(1 \leq j \leq d), \quad z_{i}-z_{j}=0(i<j) .
$$

Regular singular equations arise naturally in many problems of analysis and geometry. One of the most remarkable instances of this is the fact that the periods of the elliptic functions satisfy the hypergeometric differential equation. I think it was Fuchs who saw that this was not a special happenstance but a general phenomenon and succeeded in proving that the period integrals of Riemann surfaces satisfy regular singular differential equations, thereby inaugurating one of the most profound aspects of our subject, namely the link between meromorphic differential equations and complex algebraic geometry through period integrals. Classical analysts also considered differential equations with doubly periodic coefficients (Lamé's equation) so that already Riemann surfaces other than $\mathbf{P}_{1}$ were beginning to enter the picture as the manifolds on which the differential equations were defined.

Fabry: irregular singularities and their ramification. Nevertheless equations which are not regular singular also arise naturally. This is typically the case when the singularities become confluent. The Fuchs criterion, which describes the allowable bounds on the orders of the poles of the coefficients of the differential equation to guarantee that it is regular singular, shows that once these limits are violated, the equation has irregular singularities. The Bessel equation, the Whittaker equation, the confluent hypergeometric equation, the Airy equation, and many others are examples of differential equations with rational coefficients but with irregular singularities. From the beginning it became clear that the theory of these equations introduced new phenomena that were far different from those of the regular singular theory. For example, it is the case that for a regular singular equation, any formal solution is necessarily convergent. But this is most definitely not the case for irregular singular equations. The solutions no longer have polynomial growth at the singular point (assuming it to be $\infty$ ). Thus the equation $d u / d z=u$ has $u=e^{z}$ as its solution and this blows up exponentially at infinity. Moreover, and this was a matter of considerable surprise when the theory was being developed, it was not an easy matter to write down a full set of formal solutions to a given equation. Let us suppose the singularity to be $z=0$ and write the equation as a first order system

$$
\frac{d u}{d z}=A(z) u \quad u=\left(u_{i}\right)_{1 \leq i \leq n} \quad A=\left(a_{i j}\right)_{1 \leq i, j \leq n},
$$

where the $a_{i j}$ are meromorphic at 0 . The classical criterion for regular singularity (sufficient for systems but not necessary) is that the matrix $A$ has at most a simple pole at $z=0$. So let us suppose that the order of $A$ at 0 is $\leq-2$ and write its Taylor expansion as

$$
A=A_{r} z^{r}+A_{r+1} z^{r+1}+\ldots \quad\left(r \leq-2, A_{p} \in \mathfrak{g l}(n, \mathbf{C}), A_{r} \neq 0\right) .
$$


If the matrix $A_{r}$ has $n$ distinct eigenvalues, one can write down $n$ linearly independent formal solutions. But if the matrix $A_{r}$ is degenerate, this is no longer possible. Fabry discovered in 1885 [15(a)] that one has to go over to a covering plane, namely to work with the variable $\zeta=z^{1 / b}$ for some integer $b \geq 2$ and use meromorphic changes of the dependent variable to generate formal solutions. Moreover, the formal solutions were almost always totally divergent, namely had radius of convergence 0 . Thus there were two questions that framed themselves automatically for the irregular singular equations : how are the formal solutions determined, and what is their meaning when they are totally divergent?

Shearing transformations. The problem of finding the formal fundamental matrix of the equation $(*)$ may be looked at as the problem of finding meromorphic transformations $v=g u$ so that the equation for $v$, which is

$$
\frac{d v}{d z}=B(z) v \quad B=g A g^{-1}+\frac{d g}{d z} g^{-1},
$$

has the property that $B$ is sufficiently simple so that one can solve it directly and explicitly. The matrix $g$ need only be formally meromorphic, i.e., it should have a formal Laurent expansion

$$
g=g_{m} z^{m}+g_{m+1} z^{m+1}+\ldots,
$$

and the same should be true for $g^{-1}$, or, what amounts to the same thing, for $\operatorname{det} g$. For example, if we take

$$
A=\left(\begin{array}{ccc}
0 & 0 & 0 \\
1 & 0 & 0 \\
0 & 1 & 0
\end{array}\right) z^{-2}+\left(\begin{array}{ccc}
0 & 0 & a \\
0 & 0 & 0 \\
0 & 0 & 0
\end{array}\right) z^{-1} \quad(a \neq 0)
$$

the transformation

$$
g=\left(\begin{array}{ccc}
z^{-2 / 3} & 0 & 0 \\
0 & 0 & 0 \\
0 & 0 & z^{2 / 3}
\end{array}\right)
$$

leads us to

$$
B=\left(\begin{array}{ccc}
0 & 0 & a \\
1 & 0 & 0 \\
0 & 1 & 0
\end{array}\right) z^{-5 / 3}+\left(\begin{array}{ccc}
-1 / 3 & 0 & 0 \\
0 & 0 & 0 \\
0 & 0 & 1 / 3
\end{array}\right) z^{-1} .
$$

The leading coefficient matrix of $B$ has three distinct eigenvalues, namely the three cube roots of $-a$. This determines at once the formal fundamental matrix as a function of $\zeta=z^{1 / 3}$. This is the phenomenon that was first discovered by Fabry. The equation is then said to be ramified. It is now not difficult to see that by going over to equations of higher order one can arrange matters so that several such transformations are needed before the new equations become simple. Thus we have a structure which has several levels. The process of finding the transformations $g$ is the reduction theory of differential equations; it is formal or analytic according to whether the transformation is formally or analytically meromorphic at the singularity.

It is of course not at all obvious how the transformation $g$ was written down. Notice that conjugation by $g$ operates on each coefficient $A_{k} z^{k}$ of $A$ in two opposite 
directions, sending some entries of the matrix to those of a higher degree in $z$ and others to ones with a lower degree in $z$. For this reason the transformation $g$ was classically known as a shearing transformation. Notice that in the above example, the entry $a$ of the coefficient of $z^{-1}$ in $A$ has moved over to be an entry in the leading coefficient of $B$, as a result of which the leading coefficient of $B$ has now three distinct eigenvalues! Formal reduction theory is essentially that of discovering the sequence of appropriate shearing transformation at each level so that in the end one has an equation that is as simple as possible.

The reduction procedure is roughly as follows. One first "decouples" the system of equations along the spectral subspaces of the leading coefficient matrix, and so one comes down to the case when this coefficient matrix has a single eigenvalue, or, what amounts to the same, is nilpotent. One then determines the precise ramification of this system and makes the transformation to the new variable $\zeta=z^{1 / b}$. The procedure is repeated again at this higher level. The difficulty is in showing that the process terminates after a finite number of steps. The problem is that in principle there is nothing a priori to prevent one from encountering nilpotent leading coefficients at every level, and one has to show that this cannot happen. In the example given above, the leading coefficient is nilpotent in the first level; after the shearing transformation is applied, the leading coefficient at the second level has three distinct eigenvalues.

Poincaré: asymptotic analysis at irregular singularities and the Stokes phenomenon. The beginning of the understanding of the relationship of the formal theory to the analytic one goes back to Poincaré when he discovered in 1886 $[15(\mathrm{~b})]$ that the formal solutions written down a year earlier by Fabry are asymptotic expansions of analytic solutions; the analytic solutions are however not meromorphic, are defined only on sectors with $z=0$ as a vertex and with a sufficiently small angle, and are not unique. Indeed, as one enlarges the sector, any analytic solution asymptotic to a given formal one will cease to have this asymptotic property when the boundary of the expanding sector crosses certain lines, the so-called Stokes lines. This is what is known as the Stokes phenomenon. As the sector rotates around the origin, the requirement that the analytic solution remain asymptotic to the same formal solution forces the analytic solution to change. In terms of fundamental solutions, this means multiplying it by a constant invertible matrix. These constant matrices are the so-called Stokes multipliers. For example, for the Bessel equation (with the irregular singularity at $\infty$ ), one can write down a fundamental matrix with a well-defined asymptotic expansion on each of the two sectors, $\{0<\theta<2 \pi\}$ and $\{-\pi<\theta<\pi\}$. On the overlap the two fundamental matrices, say $F_{1}$ and $F_{2}$, are not (cannot be) the same; they are related by a constant invertible matrix $S$ satisfying $F_{1}=F_{2} S$. The matrix $S$ is the Stokes multiplier and this is the Stokes phenomenon for the Bessel equation. One can see this even more simply in the equation $d u / d z=z^{-2} u$; the solution $u=\exp (-1 / z)$ is flat in the sector $\{-\pi / 2<\theta<\pi / 2\}$, i.e., has 0 as its asymptotic expansion at $z=0$, but does not have an asymptotic expansion at $z=0$ on any larger sector. The lines $\theta= \pm \pi / 2$ which determine the boundary of this sector are the Stokes lines.

Hukuhara: Stokes lines. In the decades following Poincaré's discovery, the asymptotic theory and the related Stokes phenomenon were studied exhaustively by Trjitzinsky, Malmquist, and most decisively, by Hukuhara. Hukuhara discovered 
the correct definition of the Stokes lines in the most general case and obtained the theorem that analytic equivalence above a formal one is possible on sectors with small enough angles (see [8(c)] for these and additional references). The asymptotic expansions of the classical special functions (Airy, Bessel, Hankel, Kummer, Whittaker, etc), when interpreted as the formal solutions to these differential equations, illustrate the general theory very well; the irregular singularity is at $z=\infty$ and the expansions are valid in sectors, but different asymptotic expansions are needed in different sectors, which is the Stokes phenomenon in these cases (see [18(a)]). The Stokes phenomenon was thus seen to be a deep-lying characteristic of the given differential equation, and the classical calculations carry a clear suggestion that it captures a substantial part of the analytic structure of the solutions of the given equation.

This discussion should make it clear that reduction theory operates on two distinct levels; the formal and the analytical. The formal theory is essentially algebraic and may be regarded as the "shadow" of the analytic theory which is related to the formal one via asymptotic expansions. Thus the Poincaré asymptotic existence theorem in its general form (which was discovered only much later by the efforts of Hukuhara and others) can be formulated as the assertion that corresponding to a formal transformation

$$
A \longrightarrow B=\hat{g} a \hat{g}^{-1}+\frac{d \hat{g}}{d z} \hat{g}^{-1},
$$

there is, on sectors $\Gamma$ with sufficiently small openings, an analytic transformation

$$
A \longrightarrow B=g a g^{-1}+\frac{d g}{d z} g^{-1}
$$

with

$$
g \sim \hat{g} \text { on } \Gamma .
$$

Here $A$ and $B$ are both analytically meromorphic. However $g$ generally depends on $\Gamma$ and this dependence illustrates the Stokes phenomenon in its general formulation.

The essential presence of functions with exponential growth at the singularities in the theory of irregular singular equations suggests clearly that its foundations cannot be regarded as fully understood until one has an understanding of the structure of such analytic functions. It was E. Borel who started the first attack on this problem and showed how one can give meaning to divergent series by using the method of Borel summability, and used it to determine in a canonical manner the analytic solutions asymptotic to formal ones, at least in a number of important cases.

\section{THE MODERN FRAMEWORKS AND FORMALISMS}

I shall now give a brief discussion of the modern frameworks in which the themes described above may be viewed.

Flat connections on holomorphic vector bundles. Let me begin with the geometric point of view. The basic fact of the classical theory of ordinary differential equations is that at each point of $X:=\mathbf{P}_{1} \backslash F$ ( $F$ is the finite set of singularities of the equation) one has a finite dimensional vector space of "initial data". We put these data at the various points together and form a holomorphic vector bundle $\mathcal{V}$ 
on $X$. Moreover given any point $x$ of $X$ and any point $u$ in the fiber $\mathcal{V}_{x}$ at $x$, there is a uniquely determined section of the bundle in a neighborhood of $x$; this is the local solution with initial data $u$. In other words, we have what the geometers call a flat connection on $\mathcal{V}$. The horizontal sections of this connection are the solutions of our differential equation. Note that in this formulation one can have any Riemann surface as the base manifold. To maintain contact with the classical theory one takes $X$ to be $Y \backslash F$ where $Y$ is a compact Riemann surface. It is possible via a technical argument to assume that the bundle extends holomorphically to all of $Y$; the connection however is holomorphic only on $X$ but is meromorphic at the points of $F$. If $Y$ is $\mathbf{P}_{1}$ and the bundle is trivial, one gets the system

$$
\frac{d u}{d z}=A u
$$

where $A$ is a matrix of rational functions with singularities only in $F$; the covariant derivative associated with the connection is given by

$$
\nabla_{d / d z}=\frac{d}{d z}-A
$$

The condition for the horizontality of the section $u$ is

$$
\nabla_{d / d z} u=0 \Longleftrightarrow \frac{d u}{d z}=A u
$$

The transformation law

$$
A \longrightarrow B=g a g^{-1}+\frac{d g}{d z} g^{-1}
$$

encountered earlier is now seen to be the transformation law of connections, so that the matrices $g$ are gauge transformations.

This framework is also adequate to deal with the generalizations to several variables. Now $X$ is the manifold $Y \backslash F$ where $Y$ is compact and $F$ is a divisor of codimension 1 in $Y$. We have a holomorphic vector bundle $\mathcal{V}$ on $Y$ and a meromorphic connection $\nabla$ on $X$. But now one has to assume that this connection is flat; flatness is automatic in dimension 1 but not so in higher dimensions. The monograph of Deligne [9(a)] and the book [11] are the most detailed sources for this point of view.

Differential modules, connection matrices, and the gauge adjoint group. There is also a more algebraic framework for treating at least some of the questions arising in this context, which goes back to Manin's famous and influential paper $[10(a)]$. Let us first consider only the local structure of the differential equations near an isolated singularity which may be taken to be $z=0$. Using the usual technique, one may pass from scalar equations of order $n$ to first order systems of the form

$$
\frac{d u}{d z}=A(z) u \quad u=\left(u_{i}\right)_{1 \leq i \leq n} \quad A=\left(a_{i j}\right)_{1 \leq i, j \leq n}
$$

where the $a_{i j}$ are meromorphic at 0 . Let $\mathbf{C}_{z}$ be the field of formal Laurent series with complex coefficients containing only finitely many negative powers of $z$ and 
$\mathbf{C}_{z, \text { cgt }}$ the subfield of convergent Laurent series. Then $A$ above may be viewed as an element of the Lie algebra $\mathfrak{g l}\left(n, \mathbf{C}_{z, \mathrm{cgt}}\right)$ and the set of systems $(S)$ may be identified with the Lie algebra $\mathfrak{g l}\left(n, \mathbf{C}_{z, \text { cgt }}\right)$. If we change $u$ to $u^{\prime}=g u$ where $g \in G L\left(n, \mathbf{C}_{z, \text { cgt }}\right)$, the system $(S)$ for $u$ goes into the same type of system for $u^{\prime}$ with $A$ replaced by $A^{\prime}$ where

$$
A^{\prime}=g A g^{-1}+\frac{d g}{d z} g^{-1}=: g[A]
$$

This is of course precisely the transformation law of connections with $g$ playing the role of a gauge transformation. The matrices $A$ may thus be referred to as connection matrices. Actually if we consider the trivial vector bundle on a disk around the origin in the $z$-plane, the matrix $A$ defines a connection by the rule

$$
\nabla_{A, d / d z}:=\frac{d}{d z}-A,
$$

and the formula $(G)$ is the transformation of $\nabla_{A}$ to $\nabla_{A^{\prime}}$ by the gauge transformation defined by $g$. We may thus view the theory as the study of germs of holomorphic vector bundles $V$ equipped with connections $\nabla$ that are meromorphic at $z=0$. The space of germs of meromorphic sections of $V$ is then a vector space over $\mathbf{C}_{z \text {,cgt }}$ of dimension $n$ and is a differential module under $\nabla$.

Thus there are at least three different formalisms for studying the problems concerning the local behavior of first order systems of differential equations:

(1) The gauge adjoint action $g, A \mapsto g[A]$ of $G L\left(n, \mathbf{C}_{z}\right)$ on $\mathfrak{g l}\left(n, \mathbf{C}_{z}\right)$ as well as that of $G L\left(n, \mathbf{C}_{z, \operatorname{cgt}}\right)$ on $\mathfrak{g l}\left(n, \mathbf{C}_{z, \operatorname{cgt}}\right)[8(\mathrm{a})]$

(2) The category of germs of holomorphic vector bundles equipped with meromorphic connections $[9(\mathrm{a})]$

(3) The category of differential modules over $\mathbf{C}_{z}$ or $\mathbf{C}_{z, \text { cgt }}[10]$ [11].

These formalisms are flexible enough to handle global problems as well. For instance, if one replaces the field $\mathbf{C}_{z, \text { cgt }}$ by a globally defined ring such as the ring of meromorphic functions on a compact Riemann surface which are holomorphic away from a fixed finite subset of the surface and works with finite modules over this ring equipped with a connection, then one obtains the algebraic formalism for treating the differential equations with meromorphic coefficients on the surface from a global point of view. We may also replace $\mathbf{C}$ by a completely arbitrary commutative ring. More precisely, for any commutative $\mathbf{C}$-algebra $R$ let us write $R_{z}=R[[z]]\left[z^{-1}\right]$. We shall view $R_{z}$ as a differential ring with respect to the derivation $d / d z$. For any integer $b \geq 1$ we write $R_{z, b}=R\left[\left[z^{1 / b}\right]\right]\left[z^{-1 / b}\right], R_{z, \infty}=\cup_{b \geq 1} R_{z, b}$. If $K$ is an algebraically closed field of characteristic $0, K_{z, \infty}$ is the algebraic closure of $K_{z}$; this is the classical theorem of Puiseux (cf. J. P. Serre, Corps locaux, Hermann, 1968, p. 76, Proposition 8, $\S 2$, Ch. 4). The systems $(S)$ and the transformations $(G)$ as well as the formalisms (1) and (3) continue to make sense if we work over $R_{z}$ and $R_{z, \infty}$ in place of $\mathbf{C}_{z}$ and $\mathbf{C}_{z, \infty}$. The relative viewpoint [8 (b)] then consists of the extension of the base from $\mathbf{C}$ to $R$; it allows us to treat families of systems $(S)$ depending analytically on one or several parameters and study their behavior under gauge transformations when the gauge transformations are also allowed to depend analytically on the same parameters, by the simple device of replacing $\mathbf{C}$ with the ring $R$ of functions on the parameter space. This point of view serves 
equally well in the analytic theory where the rings $R$ are replaced by rings of germs of analytic functions defined on sectors and possessing asymptotic expansions at the vertices of these sectors.

Formal reduction over $\mathbf{C}$ and its relation to nilpotent orbits. Most of the themes mentioned in the introduction can be treated within any of these formalisms. I shall give a brief discussion of formal reduction theory. As mentioned in the introduction the essential question is that of showing that the process of reduction described there terminates after a finite number of steps. It turns out that the nilpotents that occur at the successive levels become "better and better" in the following sense : the dimensions of their conjugacy classes (as algebraic varieties in the space of matrices) keep strictly increasing. Thus, in a finite number of steps, we must encounter a leading coefficient with at least two distinct eigenvalues. Decoupling at that level now reduces the order of the system and so the finiteness of the process becomes obvious.

This increase in the dimension of the nilpotent orbits is a consequence of the theory of algebraic group actions and shows that the subject of formal reduction is essentially group-theoretic in nature. Moreover this group-theoretic method makes it possible to extend formal reduction theory to the case when $\mathbf{C}$ is replaced by a more general integral domain, i.e., to what I have called the relative context. In particular it has become possible to prove very general results on the formal reduction of analytic families of connection matrices to canonical forms. It had not been possible to do this in the classical framework. The point is that for the analytic families of matrices that arise as the leading coefficients of analytic families of systems $(S)$, their Jordan forms may exhibit abrupt jumps when the parameter crosses certain special values. This fact had been a major obstruction in the classical approaches to reduction theory of families of differential equations. From our point of view, in which reduction theory of families is to be viewed in terms of a base change from $\mathbf{C}$ to a suitable $\mathbf{C}$-algebra $R$, the problem of reduction to canonical forms thus depends in the first approximation on understanding the structure of the orbit space of nilpotent matrices over $R$ under conjugacy with respect to $G L(n, R)$. This structure is however much deeper than over $\mathbf{C}$; this is what makes reduction theory of families of connections a much more profound question than that of individual connections. It is also clear that the theory of analytic families of differential equations will work nicely only on suitable subdomains of the parameter space. Indeed the canonical forms of such a family will in general be algebraic functions (or worse) and will be single-valued analytic functions only on suitable subdomains of the original domain; at the excluded points usually the character of the system will undergo a significant change, so that these are in some sense bifurcation points or turning points [8(b)] [18(b)]. I believe it is an important problem to understand the theory of bifurcation from this relative point of view.

The formalism of the relative point of view has already led to many results that appear to lie beyond the classical methods and has suggested a variety of questions that are entirely new. In particular it appears now that the theory of groups and their actions over "very big" function fields and rings may be of importance for questions of differential equations with parameters.

Stokes sheaf, its cohomology, and its description as an affine space. I shall conclude this section with a discussion of the analytic theory in the modern framework. The key fact is the asymptotic existence theorem asserting that to any 
formal gauge transformation one can associate an analytic gauge transformation asymptotic to it, at least in a sufficiently small sector. However this analytic gauge transformation is not unique and the attempt to track down this nonuniqueness leads us to gauge transformations that are asymptotic to 1 ("flat"). They form a sheaf of groups over the unit circle (which parametrizes the directions at 0 ). These sheaves were first introduced by Malgrange and Sibuya [28] [29] in the 1970's, and our understanding of what is behind the Stokes phenomenon is entirely due to their pioneering work which pointed out the cohomological nature of the Stokes phenomenon; indeed, their work showed that the Stokes phenomenon could be given a precise formulation in terms of the cohomology of these sheaves, and further that the Stokes phenomenon characterized completely the analytic structure of the solutions of the differential equations. To any meromorphic connection matrix $A$ one can associate the corresponding Stokes sheaf $S t(A)$ whose stalk at the point $\zeta$ of the unit circle is the group of germs of flat analytic gauge automorphisms of $A$, i.e., the group of germs of analytic invertible matrices $g$ defined on sectors around $\zeta$ such that

$$
g A g^{-1}+\frac{d g}{d z} g^{-1}=A, \quad g \sim 1
$$

The Stokes multipliers which relate the fundamental matrices on different sectors with the same asymptotic expansion may now be viewed as the obstructions to the construction of a meromorphic fundamental matrix, and so appear as 1-cocycles of the sheaf $S t(A)$. Malgrange and Sibuya showed that the first cohomology space of $\operatorname{St}(A)$ classified up to analytic meromorphic equivalence at 0 the analytically meromorphic connection matrices $B$ which are formally equivalent to $A$.

The first cohomology $H^{1}(S t(A))$ is however not a very simple object to deal with because it is the sheaf cohomology associated to a sheaf of nonabelian groups. However the requirement of formal equivalence underlying the structure and the detailed knowledge of the formal canonical forms implies that the groups of sections of this sheaf are unipotent algebraic groups. Consequently it becomes possible to give the structure of an algebraic variety on $H^{1}(S t(A))$. One may view this as the moduli space for the connections formally isomorphic to $A$ (this is only a rough description). The variety structure of the Stokes multipliers was of course already treated from the classical perspective in the papers [16]. Its description from the cohomological point of view appeared in $[8(\mathrm{c})]$. But there is more; there is in fact a scheme structure on $H^{1}(S t(A))$ as Deligne observed (cf. his letters to Malgrange, Ramis, and Varadarajan [9(b)]) because one can view the Stokes sheaf as a sheaf of unipotent group schemes over $\mathbf{C}$. These ideas paved the way for the eventual solution of the local moduli problem for linear meromorphic differential equations near an irregular singularity and the result that the cohomology of the Stokes sheaf is an affine space [16] [8(c)] [30].

Finally there are two other points of view which are also at the very heart of the questions discussed here. One is the point of view of Galois differential groups which goes back to Picard and Vessiot. It associates to a differential equation the differential field generated over the field of coefficients of the equation by the solutions of the equation. This leads to the differential Galois group which is the group of automorphisms of this differential field extension over the ground field. This is an algebraic group and it carries a lot of information about the analytic 
structure of the differential equation. In a certain sense it lies at an intermediate position between the formal and the Stokes theories, and its algebraic nature makes it a little more accessible than the general analytic theory. The second is the point of view of resurgent complex analysis pioneered by Ecalle, Ramis, and their followers. This is essentially a theory of analytic functions associated to divergent formal series and thus a summability theory whose origins lie, as I pointed out in the introduction, in the work of Borel on divergent series and (Borel) summability. I have given the appropriate references in the later parts of this article but I have not made an attempt to go into any details.

\section{Structure and Formal Reduction of a CONnection over $\mathbf{C}$}

The most basic type of singularity is the so called regular singular point; it was the first to be studied historically (by Riemann and Fuchs), and moreover many irregular singularities may be obtained from the regular singularities by a limiting process (confluence). Connections whose only singularities are regular are often called Fuchsian.

Regular singular connections. The classical definition of a regular singular point for a scalar differential equation goes back to Riemann and Fuchs and requires that the solutions of the equation in the neighborhood of the singular point (say $z=0$ ) lie in the module generated over $\mathbf{C}_{z \text {,cgt }}$ by functions of the form $z^{a}(\log z)^{b}(a \in \mathbf{C}, b \in \mathbf{Z}$, and $\geq 0)$. It is equivalent, both for scalar equations and for systems $(S)$, to the requirement that in any sector with vertex at $z=0$, all solutions (which are now single-valued on the sector) are of moderate growth at 0 , i. e., are $O\left(|z|^{-N}\right)$ for some $N \geq 0$ as $z \longrightarrow 0$. For a scalar equation

$$
y^{(n)}+a_{1} y^{(n-1)}+\cdots+a_{n} y=0
$$

we have the famous criterion of Fuchs: the point $z=0$ is regular singular if and only if

$$
\text { ord }\left(a_{i}\right) \geq-i \quad(1 \leq i \leq n)
$$

where ord is the order function at 0 . For systems $(S)$ no such criterion exists; the most well-known result is the following sufficient condition going back to Sauvage: $(S)$ has 0 as a regular singular point if 0 is a simple singularity, namely if 0 is at most a simple pole for $A$ :

$$
\text { ord }(A) \geq-1 \text {. }
$$

This condition is of course not invariant under $G L\left(n, \mathbf{C}_{z, \mathrm{cgt}}\right)$. Classically, such connections $A$ are said to be of the first kind.

In the language of the gauge adjoint action $(S) A$ is regular (at $z=0$ ) if there is a constant matrix $C$ and a gauge transformation $g \in G L\left(n, \mathbf{C}_{z}\right)$ such that

$$
g[A]=z^{-1} C .
$$

If $A \in \mathfrak{g l}\left(n, \mathbf{C}_{z, \operatorname{cgt}}\right)$, then $g$ will automatically be in $G L\left(n, \mathbf{C}_{z, \text { cgt }}\right)$; this fact makes it clear that in the regular case, locally, there is no distinction between the formal and the convergent theories. The $G L(n, \mathbf{C})$-conjugacy class of $e^{2 \pi i C}$ is then uniquely determined and is the local monodromy of $A$ at $z=0$. It is of course of interest to 
determine it explicitly at least when $A$ is simple, i.e., ord $(A) \geq-1$. Let us write $A$ as a Laurent series

$$
A=A_{-1} z^{-1}+A_{0}+A_{1} z+\cdots+A_{m} z^{m}+\ldots
$$

Then $e^{2 \pi i C}=e^{2 \pi i A_{-1}}$ if no two eigenvalues of $A_{-1}$ differ by an integer. In all cases $e^{2 \pi i C}$ and $e^{2 \pi i A_{-1}}$ have the same characteristic polynomial. If there are pairs of eigenvalues of $A_{-1}$ differing by an integer, and if $m \geq 1$ is the maximum integer difference, then $e^{2 \pi i C}$ is completely determined by a knowledge of the Taylor coefficients $A_{-1+s}, s<m$, the bound being sharp. These are classical results going back to Birkhoff, Gantmacher, and possibly others (cf. [8 (a)]).

Riemann-Hilbert problem. The global study of Fuchsian connections also goes back to Riemann. Here the main interest is in the global monodromy and gives rise in fact to the most famous problem in the whole business. It was raised by Hilbert as the twenty-first problem in his celebrated Paris International Mathematical Congress address. As is well-known, the problem is to show that on $\mathbf{P}_{1}$, given any finite set $F$ and a representation $\pi$ of the fundamental group of $\mathbf{P}_{1} \backslash F$, there is a linear differential equation with rational coefficients with regular singularities at the points of $F$ (and no other singularities) whose monodromy representation is $\pi$. This problem has a long history with solutions under one or other conditions due to G. Birkhoff, J. A. Lappo-Danilevsky, and most definitively, to Plemelj (see [4(a)] for the references). One can formulate it on an arbitrary compact Riemann surface $X$ in place of $\mathbf{P}_{1}$. The fundamental existence theorem of H. Röhrl and P. Deligne (see [4(a)]) is that this can be done and that there is essentially only one way to do it. More precisely we consider the category of all holomorphic vector bundles $V$ on $X$ equipped with a holomorphic connection on $X \backslash F$ that is meromorphic at the points of $F$. The horizontal sections of this connection form a local system whose monodromy representation, on the fiber at a fixed point $x_{0}$ of $X$, of the fundamental group $G$ of $X \backslash F$ with base point $x_{0}$, will be denoted by $\pi(V)$. Then the theorem of Röhrl and Deligne asserts that the assignment

$$
V \longmapsto \pi(V)
$$

is a functor into the category of representations of $G$ which is an equivalence of categories. However, one has the feeling that there should be more, since the vector bundle with connection that comes out of this picture is very remote from the rational differential equations that Hilbert appeared to have had in mind. One way to make contact with the classical point of view is to remember that the morphisms in the category of vector bundles equipped with meromorphic (at $F$ ) connections defined above are bundle maps that are meromorphic at the points of $F$, so that the holomorphic class of the bundle on $\mathbf{P}_{1}$ can change under an isomorphism in the category. Thus it is natural to ask, for a given type of vector bundle, whether the connection can be realized in it. For $\mathbf{P}_{1}$ if we take the trivial bundle we get Hilbert's formulation with the understanding that by a differential equation with regular singularities we mean a system of the form

$$
\frac{d U}{d z}=A(z) U, \quad A(z)=\sum_{i} \frac{R_{i}}{z-a_{i}}
$$


the $a_{i}$ being the finite points of $F(\infty \in F)$ and $R_{i}$ are constant matrices. In this formulation one can interpret the classical solution of Plemelj as asserting that if one of the local monodromy transformatons at the points of $F$ is semisimple, there is such a system $(*)$. It was proved by Dekkers that when $n=2$ no condition is necessary (see $[4(\mathrm{a})]$ and $[4(\mathrm{c})])$.

This question (on $\mathbf{P}_{1}$ ) has been recently examined anew by Bolibruch and Kostov (independently). In 1989 Bolibruch discovered a counterexample to the problem when $n=3$. Then he and Kostov independently discovered the striking positive result that if the monodromy representation is irreducible, then the answer is always affirmative for any $n$ (see the paper : Bolibruch, A.A., "The Riemann-Hilbert problem", Russian Math. Surveys, 45:2 (1990), 1-47, as well as [5(c)] [6(a)(b)]).

Monodromy computations for regular singular differential equations with more than three singularities. The "direct problem" in this context is the computation of the monodromy groups of given Fuchsian equations. To go beyond Riemann's work means the consideration of equations with order greater than 2 and (or) number of singular points greater than 3. The study of such "generalized hypergeometric" equations goes back at least to Pochhammer. Recently the monodromy of the generalized hypergeometric function ${ }_{n} F_{n-1}$ has been computed by Beukers and Heckman [20]. Such equations have also been encountered in physics, especially in the so-called theory of "mirror symmetry", as Picard-Fuchs equations; see the papers of Candelas et al., Cadavid-Ferrara, and Morrison [21(a)(b)(c)] and the references in these papers.

Moduli. The vector bundle formulation of the Riemann-Hilbert problem suggests naturally the question of studying the moduli of Fuchsian connections on punctured Riemann surfaces such as $\mathbf{P}_{1} \backslash F$ where $F$ is a finite set. The number of parameters needed to parametrize Fuchsian connections with specified monodromy data was already of concern to Riemann, and the deformation theory of Fuchsian equations was considered by Schlesinger, who derived his famous equations for isomonodromic variation at the turn of the century. Indeed, for the equations

$$
\frac{d Y}{d z}=\sum_{1 \leq i \leq m} \frac{C_{j}(z)}{z-a_{j}} \quad \text { on } \mathbf{P}_{1},
$$

the deformation of the $a_{j}$ and $C_{j}$ so that the monodromy does not change leads to the Schlesinger system of completely integrable nonlinear partial differential equations for the $C_{j}$ given by

$$
\begin{aligned}
& \frac{\partial C_{i}}{\partial a_{j}}=\frac{\left[C_{i}, C_{j}\right]}{a_{j}-a_{i}} \quad(i \neq j), \\
& \frac{\partial C_{i}}{\partial a_{i}}=-\sum_{k \neq i} \frac{\left[C_{k}, C_{i}\right]}{a_{k}-a_{i}} .
\end{aligned}
$$

These questions were reexamined starting in the 1970's. For example, in a series of papers, Kazuo Okamoto studied the isomonodromic deformations on elliptic curves. Then in the 1980's Miwa, Mori, Jimbo, and Sato discovered a remarkable connection between these questions and certain problems of mathematical physics 
as well as with the theory of certain transcendental functions that were studied in the last century by Painlevé. The subject has now become very exciting and active. I refer the reader to the papers of Iwasaki $[6(\mathrm{~d})(\mathrm{e})]$ and the book $[6(\mathrm{c})]$ by Iwasaki et al. for a thorough discussion of these problems and comprehensive references for the works mentioned above. For a different treatment of the moduli problem for differential equations, Fuchsian or not, on the Riemann sphere, see M. F. Singer $[6(\mathrm{~g})]$.

Several variables. Throughout this paper I have restricted myself to ordinary differential equations. The theory has a rather far-reaching generalization in several variables. Indeed, hypergeometric functions in two variables were introduced by Appell and Picard towards the end of the last century. In [9(a)] Deligne introduced the global point of view, formulated and proved the theorem establishing a Riemann-Hilbert correspondence between monodromy groups and Fuchsian systems of integrable partial differential equations (=flat connections) on complex manifolds, and gave a treatment of the theorem of Griffiths on the regular singularity of the Gauss-Manin or Picard-Fuchs system of differential equations. Somewhat earlier, such equations were encountered and studied by Harish-Chandra in his work on the harmonic analysis on homogeneous spaces for semisimple Lie groups [22]. In recent years, Gel'fand and his coworkers have developed a general theory of hypergeometric functions in an arbitrary number of variables (see [23] for the references to this work; see also the recent book of M. Yoshida [24] and the papers of G. J. Heckman and E. M. Opdam [25]). For a proof of the basic local theorem in several variables see $[4(\mathrm{~b})]$.

Formal structure at an irregular singularity. The formal structure of a connection at an irregular singularity is more subtle even though the formal invariants do not completely determine the system $(S)$ up to meromorphic equivalence. Let us proceed to discuss it now in an appropriate context.

Let $K$ be an algebraically closed field of characteristic 0 . The complete description of the space of $G L\left(n, K_{z, \infty}\right)$-orbits in $\mathfrak{g l}\left(n, K_{z, \infty}\right)$ is classical and was the achievement of Hukuhara, Turrittin, Levelt [12] [13] [14]. To describe it we need the notion of a canonical form which is an element of $\mathfrak{g l}\left(n, K_{z, \infty}\right)$ of the form

$$
B=D_{1} z^{r_{1}}+\cdots+D_{m} z^{r_{m}}+z^{-1} C
$$

where $r_{1}<\cdots<r_{m}<-1$ are rational numbers, $C, D_{1}, \ldots, D_{m}$ are elements of $\mathfrak{g l}(n, K)$ which commute with each other, and the $D_{j}$ are nonzero and diagonal if $m \neq 0$, with the convention that $B=z^{-1} C$ if $m=0$ (the regular singular case). The $r_{j}$ are called the canonical levels, $r_{1}$ is the principal level or the Katz invariant; the connection obtained by omitting the term $z^{-1} C$ is the irregular part of $B$; the canonical form is said to be unramified if all the $r_{j}$ are integers, otherwise ramified; it is said to be defined over $K_{z, b}$ if the $r_{j}$ are in $\mathbf{Z} b^{-1}$. The smallest of the integers $b \geq 1$ with this property is called the ramification index of the canonical form $B$; in this case $B$ is said to be $\mathbf{b}$-reduced if further $0 \leq \Re \lambda<1 / b$ for all eigenvalues $\lambda$ of $C$.

One way to understand the classical theory is to view it as the identification of the orbit space of the connections under the gauge adjoint action with an orbit space for a linear action of $G L(n, K)$, i.e., a reduction from $K_{z, \infty}$ to its residue field, namely $K$. The fundamental results of Hukuhara, Levelt, and Turrittin may then be summarized as follows. 
Theorem 1. Let $K$ be an algebraically closed field of characteristic 0 . Then any $A$ in $\mathfrak{g l}\left(n, K_{z, \infty}\right)$ is equivalent under $G L\left(n, K_{z, \infty}\right)$ to a canonical form whose canonical levels depend only on the $G L\left(n, K_{z, \infty}\right)$-orbit of $A$ (and so are called the canonical levels of $A)$. If $A$ is in $\mathfrak{g l}\left(n, K_{z}\right)$ and $b$ is the ramification index of the canonical form to which $A$ is equivalent, then we can arrange matters such that for a suitable $y \in G L\left(n, K_{z, b}\right), y[A]=B$ where $B$ is a b-reduced canonical form. If

$$
B=D_{1} z^{r_{1}}+\cdots+D_{m} z^{r_{m}}+z^{-1} C, \quad B^{\prime}=D_{1}^{\prime} z^{r_{1}}+\cdots+D_{m}^{\prime} z^{r_{m}}+z^{-1} C^{\prime}
$$

are two b-reduced canonical forms over $K_{z, b}$ with the same determinations of the $z^{r_{j}}$, then they are equivalent under $G L\left(n, K_{z, b}\right)$ if and only if there is a $t \in G L(n, K)$ such that $t B t^{-1}=B^{\prime}$, i.e.,

$$
t D_{j} t^{-1}=D_{j}^{\prime}(1 \leq j \leq m), \quad t C t^{-1}=C^{\prime} .
$$

Finally, if $A$ is in $\mathfrak{g l}\left(n, K_{z}\right)$, its canonical levels are of the form $p / q$ where $p$ and $q$ are integers with $1 \leq q \leq n$, so that its canonical forms are defined over $K_{z, n !}$ and are $n$ !-reduced, and the gauge transformation taking $A$ to its canonical form may be chosen to be in $G L\left(n, K_{z, n !}\right)$.

The last statement is strictly speaking a result from the theory of connections over $K_{z}$ and is a natural consequence of the more refined theory of equivalence under $G L\left(n, K_{z}\right)$; I shall make a few brief comments on it later. It must also be noted that even if a connection is defined over $K_{z}$, its ramification index can be strictly bigger than 1; this was the discovery of Fabry in his Paris thesis of 1885 [15(a)], and may be regarded as the real beginning of formal reduction theory of connections at an irregular singularity.

The smallest of the canonical levels, namely $r_{1}$, is called the principal level. It is also called the Katz invariant because it was first highlighted by N. Katz in his beautiful paper $[10(b)]$ in which he gave arithmetic proofs of the main results of the theory of the Picard-Fuchs differential equations.

The reduction to canonical forms has been worked over by many people and so I shall only comment on some of the new aspects that have come up recently. One point on which the classical treatments were opaque is the elucidation of the reasons as to why the reduction process ends in a finite number of steps. The point is that one has to go through a succession of finite extensions $K$, and as there is no obvious way to get an a priori bound for the denominators of the levels (the bound stated in the theorem is a consequence of the reduction theory), it is not at all obvious why the process terminates. It was in trying to understand this aspect of the classical theory that Babbitt and I came up with an entirely different approach [8(a)] to the reduction problem in which the finiteness of the reduction process as well as the transition from $K_{z}$ or $K_{z, \infty}$ to the residue field $K$ are both very transparent; furthermore this new approach miraculously turned out to be also very well suited to the situation where $\mathbf{C}$ is replaced by a more general $\mathbf{C}$-algebra $R$ [8(b)].

The essence of the new approach can be explained very simply. Let us write $A$ as a Laurent series, assuming as we may that only integer powers of $z$ occur, and that $A$ has trace 0 :

$$
A=A_{r} z^{r}+A_{r+1} z^{r+1}+\ldots, \quad A_{r} \neq 0, \quad \operatorname{tr}\left(A_{r+m}\right)=0 \quad(m \geq 0) .
$$


If $A_{r}$ has two distinct eigenvalues, one can split the connection matrix $A$ as a direct sum along the spectral subspaces of $A_{r}$, and so an induction on $n$ is available. In the contrary case we may assume that $A_{r}$ is nilpotent. It is this case that is at the heart of reduction theory. Let $O$ be the $G L(n, K)$-orbit of $A_{r}$. The central step in our method is to construct a gauge transformation $g \in G L\left(n, K_{z, b}\right)$ for a suitable integer $b \geq 1$ so that the leading term $A_{r^{\prime}}^{\prime}$ of $A^{\prime}=g[A]$ satisfies one of the two following:

(1) $A_{r^{\prime}}^{\prime}$ has two distinct eigenvalues

(2) $A_{r^{\prime}}^{\prime}$ is nilpotent but lies in an affine subspace of $\mathfrak{g l}(n, K)$ that contains $A_{r}$ and is transversal to the orbit $O$; moreover, $A_{r}$ can be deformed to $A_{r^{\prime}}^{\prime}$ along this affine subspace while staying nilpotent throughout the deformation.

It can now be proved that the transversality and deformation properties described in (2) above imply that for the orbit $O^{\prime}$ of $A_{r^{\prime}}^{\prime}$ under $G L(n, K)$ we have

$$
\operatorname{dim}_{K} O^{\prime}>\operatorname{dim}_{K} O .
$$

This inequality makes it clear that the reduction process terminates in a finite number of steps and that it will end with $A$ being in canonical form. The result that the orbital dimension of a nilpotent matrix increases when it is moved transversally is to be expected of course, but its proof is not entirely trivial.

The construction of the gauge transformation $g$ above proceeds by first imbedding $A_{r}$ in a 3-dimensional simple Lie algebra by the use of the Theorem of Jacobson-Morozov according to which we can find elements $H$ and $X$ in $\mathfrak{g l}(n, K)$ such that, writing $Y=A_{r}$, we have the standard commutation rules

$$
[H, X]=2 X, \quad[H, Y]=-2 Y, \quad[X, Y]=H .
$$

The gauge transformation is then of the form $z^{q H}$ where $q$ is a suitable rational number, and the fact that $A^{\prime}$ has the stated properties is a consequence of the representation theory of the 3-dimensional algebra spanned by $H, X, Y$ acting on $\mathfrak{g l}(n, K)$ by the adjoint representation. It is not difficult to see that $z^{q H}$ is essentially the "shearing transformation" occurring in Turrittin's work [13].

It turns out that the rational number $q$ in the gauge transformation $z^{q H}$ can be explicitly determined and depends only on the Taylor coefficients $A_{r+m}$ for $0 \leq m<n(|r|-1)$. A careful examination of this and the other stages of the reduction process shows that the reduction by this method is essentially constructive and leads to the following result ([8(a)], Theorem 6.6):

Theorem 2. Let $r$ be a rational number $<-1$ and $A \in \mathfrak{g l}\left(n, \mathbf{C}_{z, \infty}\right)$ a connection of order $r$, and let $M=n(|r|-1)$. If $B \in \mathfrak{g l}\left(n, \mathbf{C}_{z, \infty}\right)$ and $A \equiv B\left(\bmod z^{M}\right)$, then the irregular parts of the canonical forms of $A$ and $B$ are the same (possibly $0)$. Moreover we can find a rational number $k \geq 0$ depending only on the $A_{r+s}$, $0 \leq s \leq M$ such that if $B \in \mathfrak{g l}\left(n, \mathbf{C}_{z, \infty}\right)$ and $A \equiv B\left(\bmod z^{M+k}\right)$, then $A$ and $B$ are equivalent under $G L\left(n, \mathbf{C}_{z, \infty}\right)$.

The bound $M=n(|r|-1)$ needed for the above theorem is sharp. To see this it is enough to consider the following example. Let $E_{i j}$ denote as usual the matrix units in the algebra of $n \times n$ matrices and let $Y_{n}=E_{21}+E_{32}+\cdots+E_{n, n-1}$; let

$$
A=z^{r} Y_{n}, \quad B=z^{r} Y_{n}+z^{r+M-1} E_{1 n} .
$$

Then $A$ is regular but $B$ has principal level $-1-1 / n$.

I shall end this review of classical reduction theory with some remarks on a few topics which I do not treat in detail here. 
Reduction theory over $\mathbf{C}_{z}$. One refinement of classical reduction theory is to determine the canonical forms for a connection defined over $\mathbf{C}_{z}$ with respect to just the gauge group over $\mathbf{C}_{z}$ and not over $\mathbf{C}_{z, \infty}$ (see [16(c)] for this and other variations). The canonical form under the full gauge group is of course an invariant, but to this one should obviously add the Galois descent data that encode the fact that the connection is defined over $\mathbf{C}_{z}$. Given $A \in \mathfrak{g l}\left(n, \mathbf{C}_{z}\right)$ we define the ramification index of $A$ as the smallest of the integers $b$ such that all its canonical levels are in $\mathbf{Z} b^{-1}$; the key fact is ([4(a)], Lemma 6.5) that if $b=b(A)$ is the ramification index of $A$, then we can find $y \in G L\left(n, \mathbf{C}_{z, b}\right)$ such that $y[A]$ is a $b$ reduced canonical form. If we now write $\Sigma$ for the joint spectrum of the $D_{j}$ and $P_{\sigma}$ $(\sigma \in \Sigma)$ for the projections on the spectral spaces indexed by $\sigma$, and write $C_{\sigma}$ for the restrictions of $C$ to these subspaces, then the facts that $A$ is defined over $\mathbf{C}_{z}$ and $B$ is $b$-reduced imply first of all that $\Sigma$ is stable under the Galois group of $\mathbf{C}_{z, b}$ over $\mathbf{C}_{z}$, and further that there is a representation $\omega$ of the Galois group such that

$$
\omega(\gamma) P_{\sigma} \omega(\gamma)^{-1}=P_{\sigma^{\gamma}}, \quad \omega(\gamma) C_{\sigma} \omega(\gamma)^{-1}=C_{\sigma^{\gamma}} .
$$

The Galois group is of course isomorphic to the group of $b^{\text {th }}$ roots of unity. The system $\left(\left(P_{\sigma}\right),\left(C_{\sigma}\right), \omega\right)$ is called the Galois descent data associated to $A$. The fundamental result is that the above procedure gives rise to a bijection of the set of isomorphism classes of descent data with the set of $G L(n, \mathbf{C})$-isomorphism classes of connections with ramification index $b$. One can construct models of canonical forms over $\mathbf{C}$ using this result; among other things such models show at once that the canonical levels are rational numbers $a / q$ where $a$ and $q$ are integers and $1 \leq q \leq n$ ([4(a)], section 6).

Connections with linear algebraic groups as structure groups. The above discussion suggests that the entire theory of connections and their reduction may be carried out with $\mathfrak{g l}(n, K)$ and $G L(n, K)$ replaced by an arbitrary algebraic Lie algebra and Lie group over $K$. This is in fact true and the classical reduction theorem formulated above remains true without any changes for connection matrices with an arbitrary affine algebraic group over $G$ as the structure group [8(a), sections $8,9]$.

I shall now describe briefly the basic definitions and a few results in this extension of the classical theory. Let $\mathbf{G}$ be a linear algebraic group defined over $\mathbf{C}$ and $\mathfrak{g}$ its Lie algebra. For any $\mathbf{C}$-algebra $R$ we write $G(R)$ (resp. $\mathfrak{g}(R)$ ) for the group (resp. Lie algebra) of $R$-points of $\mathbf{G}$ (resp. $\mathfrak{g})$. Given any integer $b \geq 1$ and any $x \in G\left(\mathbf{C}_{z, b}\right)$ there is a uniquely determined element $\delta_{\mathbf{G}}(x) \in \mathfrak{g}\left(\mathbf{C}_{z, b}\right)$ with the property that for any matrix representation (over $\mathbf{C}$ ) $\rho$ of $\mathbf{G}$,

$$
d \rho\left(\delta_{\mathbf{G}}(x)\right)=\left(\frac{d}{d z} \rho(x)\right) \rho(x)^{-1} .
$$

If $\zeta\left(\mathbf{G} \longrightarrow \mathbf{G}^{\prime}\right)$ is a $\mathbf{C}$-morphism of algebraic groups,

$$
d \zeta\left(\delta_{\mathbf{G}}(x)\right)=\delta_{\mathbf{G}^{\prime}}(\zeta(x)) \quad\left(x \in G\left(\mathbf{C}_{z, \infty}\right)\right) .
$$

If there is no need to refer to $\mathbf{G}$, we omit $\mathbf{G}$ and write $\delta$. Once $\delta$ is defined we have the gauge adjoint action of $\mathbf{G}\left(\mathbf{C}_{z, \infty}\right)$ on $\mathfrak{g}\left(\mathbf{C}_{z, \infty}\right)$ :

$$
x[A]=\operatorname{Ad}(x)(A)+\delta(x) \quad\left(x \in \mathbf{G}\left(\widehat{\mathbf{C}}_{\infty}\right), A \in \mathfrak{g}\left(\widehat{\mathbf{C}}_{\infty}\right)\right) .
$$


This is of course consistent with our earlier definitions; indeed, if $\mathbf{G}$ is already given as a matrix group, $\delta(x)=(d x / d z) x^{-1}$ and $\operatorname{Ad}(x)(A)=x A x^{-1}$. The notion of the canonical form extends in an obvious manner to this new setting; in defining canonical forms for example we must simply require that the $D_{j}$ are semisimple elements of $\mathfrak{g}$. The elements of $\mathfrak{g}\left(\mathbf{C}_{z, \infty}\right)$ are $\mathbf{G}$-connections; those in $\mathfrak{g}\left(\mathbf{C}_{z, b}\right)$ are defined over $\mathbf{C}_{z, b}$. The goal of reduction theory is to show that any $\mathbf{G}$-connection is equivalent under the gauge adjoint action of $\mathbf{G}\left(\mathbf{C}_{z, \infty}\right)$ to a canonical form and that this canonical form is essentially unique. The interesting cases are when $\mathbf{G}$ is the orthogonal or the symplectic group and the connection matrices belong to the corresponding Lie algebra.

This is proved in three steps. First one treats the case when $\mathbf{G}$ is unipotent; in this case $\mathfrak{g}$ has no nonzero semisimple elements, any canonical form is of the form $z^{-1} C$, and all connections will be regular. The next step is to extend this result, by induction on the dimension of $\mathbf{G}$, to the case when $\mathbf{G}$ is solvable. The basic case is to do this by direct calculation for the 2-dimensional group of automorphisms of the affine line. The general case, again by induction on dimension, reduces to the case when $\mathbf{G}$ is reductive. This is done exactly as the case of $\mathfrak{g l}(n)$, because the Jacobson-Morozov theorem is available still. For a full treatment I refer the reader to $[8(\mathrm{a})]$ (cf. Sections 8 and 9, especially Theorem 9.5).

Here again one may ask how many terms of the Taylor series of $A$ are needed to predict the irregular part of the canonical form of $A$. One can prove a very nice generalization of the bound $n(|r|-1)$ established in the $\mathfrak{g l}(n)$ case. For any Lie algebra $\mathfrak{g}$ let $\mathfrak{g}_{0}$ be its reductive quotient obtained by dividing by the nilradical, and let $N(\mathfrak{g})$ be the maximum of the numbers

$$
1+m_{1}+m_{2}+\cdots+m_{r}
$$

for all positive roots

$$
m_{1} \alpha_{1}+m_{2} \alpha_{2}+\cdots+m_{r} \alpha_{r}
$$

of $\mathfrak{g}_{0}$, where $\alpha_{i}(1 \leq i \leq r)$ form a simple system of roots of $\mathfrak{g}_{0}$. We have

$$
N(\mathfrak{g l}(n))=n
$$

Then (cf. Theorem 9.7 of [8(a)]) one can prove that the same theorem as in the classical case is valid with $n(|r|-1)$ replaced by

$$
N(\mathfrak{g})(|r|-1) .
$$

Formal invariants at an irregular singularity and their computation. The study of formal invariants of differential operators that are explicitly defined in terms of the coefficients has been pursued by many people. Recently R. Sommeling [26] has made a definitive study of this problem by introducing what he calls the characteristic class of a differential operator or a connection over a differential field of characteristic 0. His method contains all invariants studied so far; moreover, he gives algorithms for calculating either the canonical form (improving one such for the determination of the canonical form presented in [8(a)]) or some other invariants. 
Irregular singularities in several variables. The theory of systems in several variables with irregular singularities is not nearly so developed; one of the reasons is that there are not many studies of explicit equations and their solutions. The monograph of Majima [27(a)] develops some aspects of the asymptotic theory of such equations. Some fundamental aspects of irregular singularities from the formal point of view were studied by Levelt and van den Essen in [27(b)].

\section{Formal REDUCTION OVER MORE GENERAL RINGS}

The theory of formal reduction of connections over more general rings follows the same lines as that of individual systems. However there are new phenomena which are very interesting both conceptually and technically. Our point of view is to view reduction theory in terms of a base change from $\mathbf{C}$ to a general $\mathbf{C}$-algebra $R$. Clearly the problem depends on understanding the structure of the the orbit space of nilpotent matrices over $R$ under conjugacy with respect to $G L(n, R)$. At present it has not been possible to prove a general theorem of reduction to canonical form of an arbitrary element of $\mathfrak{g l}\left(n, R_{z}\right)$ that is as comprehensive as in the case of $\mathbf{C}$; indeed, it is not even clear what the concept of a canonical form in this generality should be. If we however retain the same definition of a canonical form as before, then one can prove that under appropriate conditions reduction to canonical forms is possible.

Let us now consider a domain $R$ and an $A \in \mathfrak{g l}\left(n, R_{z}\right)$ and view it first as an element of $\mathfrak{g l}\left(n, K_{z, \infty}\right)$ where $K$ is the algebraic closure of the quotient field $F$ of $R$. It then makes sense to speak of the canonical form of $A$ under $G L\left(n, K_{z, \infty}\right)^{-}$ equivalence. However the elements of the matrices $C, D_{j}$ that occur in the canonical form are not necessarily in $R$, not even in $F$, the quotient field of $R$, but are only algebraic over $F$. So the following definition is natural.

Definition. Let $R$ be a domain and let notation be as above. Let $A \in \mathfrak{g l}\left(n, R_{z}\right)$. We say that $A$ is well behaved if it is equivalent to a canonical form (which is also called well behaved)

$$
B=D_{1} z^{r_{1}}+\cdots+D_{m} z^{r_{m}}+z^{-1} C
$$

under $G L\left(n, K_{z, \infty}\right)$ where

(1) the $D_{j}$ are diagonal matrices with entries in $R$

(2) if $\left(\lambda_{1}, \ldots, \lambda_{m}\right)$ and $\left(\lambda_{1}^{\prime}, \ldots, \lambda_{m}^{\prime}\right)$ are distinct elements in the joint spectrum of $\left(D_{1}, \ldots, D_{m}\right)$, and if $k$ is the first of the integers $i$ for which $\lambda_{i} \neq \lambda_{i}^{\prime}$, then $\lambda_{k}-\lambda_{k}^{\prime}$ is a unit of $R$.

The concept of well behaved connection matrices is somewhat stringent. It singles out only those connections that are split over $R$ in an obvious sense. There are examples of connections that are not well behaved and which cannot be reduced to a canonical form under $G L\left(n, R_{z, \infty}\right)$.

Example. Let $R=\mathcal{O}_{1}$, the ring of convergent power series in one variable $\lambda$. Let

$$
A=\left(\begin{array}{cc}
\lambda^{p} & 0 \\
0 & -\lambda^{p}
\end{array}\right) z^{-2}+\left(\begin{array}{cc}
0 & 0 \\
1 & 0
\end{array}\right) z^{-1} \quad(p \geq 1)
$$


Then $A$ is not well behaved and is also not equivalent to any canonical form under $G L\left(2, R_{z, \infty}\right)$. Under $G L\left(2, F_{z}\right)$ it is equivalent to the canonical form

$$
\left(\begin{array}{cc}
\lambda^{p} & 0 \\
0 & -\lambda^{p}
\end{array}\right) z^{-2}
$$

Here the two eigenvalues $\pm \lambda^{p}$ differ by $2 \lambda^{p}$ which is not a unit of $R$. Notice also that for $\lambda \neq 0$ the connection is irregular of level -2 , but at $\lambda=0$ it becomes regular singular.

On the other hand the following result ([8(b)], Lemma 10.2.2) shows that well behavedness is a useful concept.

Proposition. Let $R$ be the ring of analytic functions on some fixed polydisk in $\mathbf{C}^{d}$, and let $A \in \mathfrak{g l}\left(n, R_{z}\right)$ be such that for all $\lambda$ in that polydisk the complex connection matrices $A(\lambda)$ are unramified and have the same canonical form. Then $A$ is well behaved.

For well behaved connections on suitably restricted rings one can establish a reduction to canonical form. I give here a sampling of theorems that can be proved.

Theorem 1([8(b)], Section $\mathbf{7 . 5})$. Let $R$ be a discrete valuation ring with an algebraically closed residue field of characteristic zero and let $A \in \mathfrak{g l}\left(n, R_{z, \infty}\right)$ be a well behaved connection. Then $A$ can be reduced to a weak canonical form over $R_{z, \infty}$, i.e., there exist $x \in G L\left(n, R_{z, \infty}\right)$ and a (well behaved) canonical form $B=$ $D_{1} z^{r_{1}}+\cdots+D_{m} z^{r_{m}}+z^{-1} C$ defined over $R$ such that

$$
x[A]=B+E, \quad E=\sum_{t>-1} E_{t} z^{t}, \quad\left[E_{t}, D_{j}\right]=0 \quad \text { for all } t, j .
$$

If $R$ is Henselian ${ }^{1}$ (in particular if $R=\mathcal{O}$ ), we can choose $E=0$ so that $A$ is equivalent to a canonical form over $G L\left(n, R_{z, \infty}\right)$.

Theorem 1 is essentially the 1-dimensional case of relative reduction theory. Higher dimensional results are less definitive ([8(b)], Section 8). For rings of analytic functions and function germs it is possible to extend Theorem 1 rather completely. It would be of interest to examine whether generalizations of Theorem 1 exist for wider classes of Noetherian integrally closed regular local domains.

Theorem 2 ([8(b)], Section 9). Let $R=\mathcal{O}_{d}$ and let $A \in \mathfrak{g l}\left(n, R_{z, \infty}\right)$ be a well behaved connection. Then there exist a (well behaved) canonical form $B$ and $x \in G L\left(n, R_{z, \infty}\right)$ such that $x[A]=B$.

Analytic families of connections whose canonical forms do not depend on the parameters (isoformal families, such as the Bessel or Whittaker families) are well behaved. For them we have the following result, stated in a sharper form than the preceding theorems, in terms of reduction over $R_{z}$ itself.

Theorem $3\left([\mathbf{8}(\mathbf{c})]\right.$, I, Section 1.5). Let $R=\mathcal{O}_{d}$ and let $\Delta$ be a polydisk in $\mathbf{C}^{d}$ centered at the origin. Let $A \in \mathfrak{g l}\left(n, R(\Delta)_{z}\right)$ be a connection such that $A(\lambda)$ is equivalent to $A(0)$ under $G L(n, \mathbf{C})$ for all $\lambda \in \Delta$. Then there exists a concentric polydisk $\Delta_{1} \subset \Delta$ and an $x \in G L\left(n, R\left(\Delta_{1}\right)_{z}\right)$ such that $x[A]=A(0)$.

\footnotetext{
${ }^{1}$ See M. Nagata, Local Rings, Interscience, New York, 1962, pp. 103-104, for the definition of Henselian rings.
} 


\section{Method of Proof of Theorems $4.1-4.3$}

I shall now try to give a brief outline of the ideas behind the proofs of these theorems. As usual write

$$
A=A_{r} z^{r}+A_{r+1} z^{r+1}+\ldots, \quad A_{r} \neq 0,
$$

and assume that $A$ is well behaved. One wants to imitate the proofs in the absolute situation and so begins with a study of the nilpotent orbits.

Nilpotent matrices over Noetherian domains and their orbits. Let $R$ be a Noetherian domain with quotient field $F$. The basic problem is the following: fix a $G L(n, F)$-orbit $\Omega$ of nonzero nilpotent $n \times n$ matrices and let $\Omega(R)$ be the subset of $\Omega$ of those matrices with entries in $R$. Then $G L(n, R)$ operates on $\Omega(R)$ and we want to describe the orbit space $G L(n, R) \backslash \Omega(R)$. It turns out that the orbit space is stratified by certain discrete invariants, so called because when $R$ is a discrete valuation ring these are essentially certain sets of integers. Namely, one can define an ordered sequence of torsion $R$-modules $M_{j}(\omega)$ associated to any $G L(n, R)$-orbit $\omega$ in $\Omega(R)$ ([8(b)], p. 16). Indeed, associated to any $L \in \Omega(R)$ there is a canonical filtration (the "Hodge" filtration) in $R^{n}$, say $\left(W_{t}\right)_{t \in \mathbf{Z}}$, such that

(i) $W_{t}=0$ for $t<<0$ and $W_{t}=R^{n}$ for $t>>0$

(ii) $W_{s} \subset W_{t}$ if $s<t$

(iii) $L W_{t} \subset W_{t-2}$ for all $t$

(iv) If we write $\bar{W}_{t}=W_{t} / W_{t-1}$, then for all $j \geq 0$, the maps $L_{j}$ induced by $L^{j}$ :

$$
L_{j}: \bar{W}_{j} \longrightarrow \bar{W}_{-j}
$$

are of full rank.

Then

$$
M_{j}:=\bar{W}_{-j} / L_{j}\left(\bar{W}_{j}\right)
$$

are finite torsion $R$-modules and their isomorphism classes are invariants for the $G L(n, R)$-orbit of $L$. One can then ask two basic questions.

(1) Fix finite torsion modules $S_{j}$ and let $\Omega\left(R:\left(S_{j}\right)\right)$ be the space of orbits $\omega \subset \Omega(R)$ for which $M_{j}(\omega) \simeq S_{j}$ for all $j$. What is the structure of $\Omega\left(R:\left(S_{j}\right)\right)$ ?

(2) Given analytic family of nilpotents $L(\varepsilon)$ with $L(\varepsilon) \in \Omega\left(R:\left(S_{j}\right)\right)$ for $\varepsilon \neq 0$, how do the invariants $M_{j}$ change when $\varepsilon=0$ ?

It appears difficult to say much about these questions for general $R$. However when $R$ is a discrete valuation ring, one can say a little more. Let us therefore make this assumption from now on. For small values of $n$, with $R=\mathcal{O}$, one can explicitly calculate the orbit spaces $\Omega\left(R:\left(M_{j}\right)\right)$ and find that they are finite dimensional constructible sets. But the structure of these orbit spaces for arbitrary $n$ is somewhat more complicated [17]. Fortunately for applications to reduction theory of connections over $R$ it does not appear to be necessary to know the structure of these orbit spaces in great detail. Let us write for any nilpotent $L, d_{0}(L)$ for the codimension of the orbit $\Omega$ and $d_{j}(L)$ for the degree of the $R$-module $M_{j}$. In this manner we have a vector $\mathbf{d}(L)$ defined by

$$
\mathbf{d}=\mathbf{d}(L)=\left(d_{0}(L), d_{1}(L), \ldots\right) .
$$


The $d_{j}$ are integers $\geq 0$ and vanishing for all $j \geq J$ where $J$ depends only on the orbit $\Omega$. We regard $\mathbf{d}(L)$ as an element of $\mathbf{Z}^{J}$, the latter being given its natural lexicographic ordering. The vector $d(L)$ is an invariant of the $G L(n, R)$-orbit of $L$, and the central result for applications to reduction theory is the following ([8(b)], Theorems 4.2.4, 5.2.3, and 5.4.1):

Semicontinuity Theorem. If $L$ is admissible and is moved strictly transversally within the class of nilpotents over $R$ to a nilpotent $L^{\prime}$, then either the orbital dimension of $L^{\prime}$ over the field $F$ is strictly bigger than that of $L$, or else, the nilpotents stay in the same orbit over $F$ but the vector $\mathbf{d}\left(L^{\prime}\right)$ is strictly less than the vector $\mathbf{d}(L)$ in the lexicographic ordering of $\mathbf{Z}^{J}$. We denote this situation by

$$
L^{\prime} \prec \prec L \text {. }
$$

Let me now explain what admissibility and strict transversality mean in this context. First of all, admissibility means roughly that half of the Jacobson-Morozov theorem is true. More precisely, we have the following:

Definition. Let $H$ be an endomorphism of $R^{n}$. We say $H$ is associated to $L$ if the following is true:

(i) $H$ is semisimple over $F$ with only integral eigenvalues

(ii) If $W^{(t)}$ is the submodule of $R^{n}$ defined by $W^{(t)}=H(t) \cap R^{n}$ where $H(t)$ is the eigenspace in $F^{n}$ of $H$ for the eigenvalue $t$, then $R^{n}=\oplus_{t \in \mathbf{Z}} W^{(t)}$

(iii) For all $t \in \mathbf{Z}, W_{t}=\oplus_{s \leq t} W^{(s)},\left(W_{t}\right)_{t \in \mathbf{Z}}$ being the canonical filtration of $L$. Then $L$ is admissible if there is an $H$ associated to $L$ such that $[H, L]=-2 L$.

Since $R$ is a discrete valuation ring the torsion free nature of the modules $W_{t} / W_{s}$ $(s<t)$ implies their freeness and thus the existence of submodules $W^{(t)}$ such that $W_{t}=W_{t-1} \oplus W^{(t)}$; the element $H \in \operatorname{End}\left(R^{n}\right)$ defined by requiring that $H u=t u$ for $u \in W^{(t)}$ has the properties (i)-(iii) above. It may not be associated to $L$; however, if we expand $L$ in terms of the eigenspaces in $\operatorname{End}\left(R^{n}\right)$ for $\operatorname{ad}(H)$, it is clear that $L=L_{-2}+L_{-3}+\ldots$ where $L_{r}$ is in $\operatorname{End}\left(R^{n}\right)$ with $\left[H, L_{r}\right]=r L_{r}$, from which it follows that $L^{\prime}:=L_{-2}$ is an admissible nilpotent such that $H$ is associated to $L^{\prime}$, and what is decisive, $L^{\prime}$ has the same filtration as $L$ and the same torsion modules $M_{j}$ ([8 (b)], Proposition 5.2.3). From the above remarks it follows that

$$
\mathbf{d}(L)=\mathbf{d}\left(L^{\prime}\right) .
$$

Thus admissibility is not such a strong demand and one can work around nonadmissibility in reduction theory.

Strict transversality is a sharpening of transversality over C. Let $S$ be $\operatorname{an} \operatorname{ad}(H)$ invariant $\mathbf{C}$-linear subspace of $\operatorname{End}\left(R^{n}\right)$ which is complementary to the range of $\operatorname{ad}(L)$, i.e., complementary to the tangent space at $L$ to the orbit of $L$, and $S^{+}$, the subspace of $S$ spanned by the eigenvectors of $\operatorname{ad}(H)$ for the eigenvalues $\geq-1$.

Definition. The family $L(\varepsilon)$ of nilpotents $(L(0)=L)$ in $\operatorname{End}\left(R^{n}\right)$ is strictly transversal if $L(\varepsilon) \in L+S^{+}$for all $\varepsilon$.

In analogy with what happens in the case of $\mathbf{C}$ it would be natural to say that the family is transversal if $L(\varepsilon) \in L+S$ for all $\varepsilon$. If $R=\mathbf{C}$ it is automatic that $S=S^{+}$ but this is not always true for general $R$, so that what we have is a sharpening of the transversality condition. 
5.1. Reduction theory of connections $A \in \mathfrak{g l}\left(n, R_{z, \infty}\right)$ when $R$ is a discrete valuation ring: Theorem 4.1. The essential case is, as in the absolute situation, the following: for some integer $r>-1$,

$$
A=A_{r} z^{r}+A_{r+1} z^{r+1}+\ldots \quad A_{r}=L \neq 0, \quad L \quad \text { is a nilpotent in } \operatorname{End}\left(R^{n}\right) .
$$

The method now is to imitate the argument in the absolute case, but before doing that we have to take into account the fact that the nilpotent $L$ may not be admissible. So one has to replace $A$ by a connection $A^{\prime}$ gauge equivalent to it under $G L\left(n, R_{z, b}\right)$ for some $b \geq 1$ with the property that $A^{\prime}$ has order $r^{\prime}, r<r^{\prime}<-1$ and that $A_{r^{\prime}}^{\prime}$, the leading coefficient of $A^{\prime}$, is an admissible nilpotent; indeed it will be the admissible nilpotent constructed above in the remarks made earlier. The transition from $A$ to $A^{\prime}$ has thus been accomplished without changing the invariant $\mathbf{d}$ of the leading coefficient of the connection. In other words we may suppose that the connection $A$ itself has an admissible leading coefficient. It is now possible to proceed as in the absolute case. If $A$ is not regular, then we can find a gauge transformation $x$ taking $A$ to $A^{\prime}$ :

$$
x=z^{q H}, \quad A^{\prime}=x[A],
$$

where $H$ is associated to $A_{r}$; the leading coefficient $A_{r^{\prime}}^{\prime}$ of $A^{\prime}$, if nilpotent, has the following property :

$$
A_{r^{\prime}}^{\prime} \neq A_{r}, \quad A_{r^{\prime}}^{\prime} \in A_{r}+S^{+} .
$$

It then follows that it can be connected to $A_{r}$ by a strictly transversal nilpotent deformation of the latter. The semicontinuity theorem would now imply that

$$
A_{r^{\prime}}^{\prime} \quad \prec \prec \quad A_{r} .
$$

A downward induction with respect to $\prec \prec$ now proves that the reduction process is finite and leads to a weak canonical form. If the ring $R$ is Henselian, one can refine this into a canonical form itself ([8 (b)], Section 7).

The proof of the semicontinuity theorem itself is somewhat involved. Let me give a brief sketch of the steps involved in its proof.

Step 1: The first step ([8(b)], Theorem 4.2.4) is to prove that for a nilpotent deformation $(L(t))$ where all the $L(t)$ are in the same orbit over the field $F$, we have, for all $t$ near 0 ,

$$
\mathbf{d}(L(t))<<\mathbf{d}(L(0))
$$

where $<<$ is the lexicographic ordering mentioned above; and further that if there is equality here for all $t$ near enough to 0 , then we can find a deformation $(g(t))$ of the identity such that $L^{\prime}(t):=g(t) L(t) g(t)^{-1}$ has the same filtration over $R$ as $L(0)$ for all $t$ sufficiently close to 0.

Step 2: The second step is to study more closely the deformation $(L(t))$ under the assumptions :

(a) We have

$$
\mathbf{d}(L(t))=\mathbf{d}(L(0))
$$

for all $t$ near 0 .

(b) The family $(L(t))$ is transversal, i.e.,

$$
L(t) \in L(0)+S
$$

for all $t$ near 0 . 
The point is now to prove that this forces the relation

$$
L(t) \in L(0)+S^{-}
$$

for all $t$ near $0([8(\mathrm{~b})]$, Theorem 5.2.3). For the applications we have in mind it is enough to work in the situation where $t$ varies in a neighborhood of 0 in the complex plane $\mathbf{C}$. Then we can write $L(t)$ and $g(t)$ (in step 1 ) as power series

$$
L(t)=L(0)+\sum_{m \geq 1} L_{m} t^{m}, \quad g(t)=I+\sum_{m \geq 1} g_{m} t^{m} .
$$

The condition that $L(0)$ and $L^{\prime}(t)=g(t) L(t) g(t)^{-1}$ have the same filtration over $R$ for all $t$ near 0 now gives identities involving the $g_{r}$ and $L_{s}$. If we assume that $L(t) \notin L(0)+S^{-}$for values of $t$ arbitrarily close to 0 , then we can consider the smallest $m \geq 1$ for which $L_{m} \notin S^{-}$, and its projection $L_{m}^{+}$in $S^{+}$, which is now nonzero. The identities mentioned above now force the result that $L_{m}^{+} \in\left[L(0), E_{R}\right]$ where $E_{R}$ is $\mathfrak{g l}(n, R)$. This is a contradiction since $0 \neq L_{m}^{+} \in S$ and $S \cap\left[L(0), E_{R}\right]=$ 0 .

Step 3: The last step is to show ([8(b)], Theorem 5.4.1) that if $0 \neq Z \in S^{+}$is such that $L(0)+Z$ is nilpotent, then

$$
L(0)+Z \prec \prec L(0) .
$$

This is done by constructing a nilpotent deformation $(L(t))$ such that $L(1)=L(0)+$ $Z$ and $L(t) \in L(0)+S^{+}$for all $t$.

5.2. Reduction theory of connections $A \in \mathfrak{g l}\left(n, R_{z, \infty}\right)$ for $R$ Noetherian and integrally closed. We now assume that $R$ is a Noetherian domain that is integrally closed. Let $F$ be its quotient field. Then one knows that for any minimal prime ideal $\mathfrak{p}$ in $R$, the localization at $\mathfrak{p}$, say $R_{\mathfrak{p}}$, is a discrete valuation ring. In view of $\mathbf{5 . 1}$ we now have a reduction to canonical form over $R_{\mathfrak{p}}$. The gauge transformation implementing this reduction is not unique, and so it is not immediately clear how to choose them so that they vary in a coherent manner as $\mathfrak{p}$ varies. To do this one goes over to the differential module associated to $A$, namely, $\left(F_{z, \infty}^{n}, \nabla_{A}\right)$ where $\nabla_{A, d / d z}=d / d z-A$. The existence of a canonical form over $F_{z, \infty}$ together with the well behavedness of $A$ leads to a unique decomposition of this module into a direct sum of differential submodules on each of which the connection $\nabla_{A}$ has a scalar irregular part. The uniqueness of this decomposition together with the existence of a reduction to canonical form over $R_{\mathfrak{p}}$ now shows that the projection operators onto the submodules in the decomposition are defined over $R_{\mathfrak{p}_{z, \infty}}$ for all $\mathfrak{p}$. As $R=\cap_{\mathfrak{p}} R_{\mathfrak{p}}$, we may conclude that they are already defined over $R_{z, \infty}$. In other words, if we write $M=R_{z, \infty}^{n}$, the differential module $\left(M, \nabla_{A}\right)$ has a unique decomposition

$$
\left(M, \nabla_{A}\right)=\oplus\left(M_{f}, \nabla_{f}\right)
$$

where the $f$ vary over the joint spectrum of the $D$ 's that occur in the canonical form of $A$, and

$$
\nabla_{f}=f \cdot 1+\nabla_{f}^{*},
$$

with the connection $\nabla_{f}^{*}$ having the property that when tensored by $F_{z, \infty}$ it is of order $\geq-1$ (of the first kind, in classical terminology). The modules $M_{j}$ are certainly projective, but they may not be free. We thus have the following result. 
Theorem 1 ([8(b)], Theorem 8.1.2). Let $R$ be a Noetherian integrally closed domain with the property that for all integers $b \geq 1$, all finite projective modules over $R_{z, b}$ are free. If $A \in \mathfrak{g l}\left(n, R_{z, \infty}\right)$ is well behaved and we write $\Sigma(A) \subset R$ for the spectrum of the irregular part of the canonical form of $A$, we can find $x \in$ $G L\left(n, R_{z, \infty}\right)$ and connections $A_{f} \in \mathfrak{g l}\left(n_{f}, R_{z, \infty}\right)(f \in \Sigma(A))$ such that

(i) $x[A]=\oplus_{f \in \Sigma(A)} A_{f}$

(ii) $A_{f}=f \cdot 1+A_{f}^{*}$ where $A_{f}^{*}$ is regular, i.e., $A_{f}^{*}$ is isomorphic, over $F_{z, \infty}$, to a connection in $\mathfrak{g l}\left(n_{f}, R_{z, \infty}\right)$ of order $\geq-1$.

In particular this is true if $R=\mathcal{O}_{d}$.

Remark. I do not know if the hypothesis on $R$ is satisfied whenever $R$ is a regular local domain (of course Noetherian and integrally closed). However, if $R=\mathcal{O}_{d}$, it can be proved that all finite projective modules over $R_{z, b}$ are free. This is of course a generalization of the Theorem of Quillen-Souslin that asserts the freeness of finite projective modules over polynomial rings. For the case of the rings of Laurent series considered here this generalization was established by Bruns, Evans, and Griffith [19] in the case when the coefficients of the Laurent series are formal power series; the case of relevance to us is one where the coefficients are in the convergent power series ring in $d$ variables, but it is not difficult to obtain a variant of their argument that will take care of this situation [8(b)] (Appendix A2, Theorem 3).

5.3. Reduction of the connection $A \in \mathfrak{g l}\left(n, R_{z, \infty}\right)$ when $R=\mathcal{O}_{d}$ or $\mathcal{O}(\Delta)$ : Theorems 4.2 and 4.3. It is now only a question of proving that if $B \in \mathfrak{g l}\left(n, R_{z}\right)$ is equivalent under $G L\left(n, F_{z}\right)$ to a connection of order $\geq-1$, then there is an endomorphism $C$ of $R^{n}$ and a $g \in G L\left(n, R_{z}\right)$ such that $g[B]=z^{-1} C$. This result, when applied to the connections $A_{f}^{*}$ now leads to Theorem 4.2. Theorem 4.3 then follows without too much difficulty from Theorem 4.2. For the details of this step the reader should look into Sections 9 and 10 of [8(b)] and I, 1.5 of [8(c)].

\section{Analytic theory. Asymptotic analysis, Stokes Phenomenon, And the Stokes sheaf}

If the system $(S)$ is irregular singular at $z=0$, its formal structure is very far from determining the analytic structure. As I have explained in the introductory sections, this is essentially a theory of asymptotic analysis at the point $z=0$. There are three aspects to this program.

1. To establish a sufficiently general asymptotic existence theorem that will allow us to associate to any formal solution of $(S)$ and any sector of angle small enough an analytic solution asymptotic to it on the sector.

2. To introduce the Stokes sheaf of a connection and show that its first cohomology classifies up to local meromorphic equivalence all connections formally equivalent to the given one.

3. To show that this first cohomology is a complex analytic variety in a natural sense and determine its structure (moduli).

Asymptotic existence theorem. I formulate this in the form given in [18(a)] using sectors $\Gamma$ with vertex at $z=0$ where

$$
\Gamma=\left\{z=r e^{i \theta} \mid r>0, a<\theta<b\right\}
$$

with $0 \leq a<b \leq 2 \pi$; if $\delta>0, \Gamma_{\delta}$ is the set of points in $\Gamma$ for which $r<\delta$. For sectors $\Gamma, \Gamma^{\prime}, \Gamma^{\prime} \subset \subset \Gamma$ means that the bounding rays of $\Gamma^{\prime}$ are within $\Gamma$. An open 
set $\Omega$ is asymptotic to the sector $\Gamma$ if $\Omega \subset \Gamma$ and for each sector $\Gamma^{\prime} \subset \subset \Gamma$ there is a $\delta>0$ such that $\Gamma_{\delta}^{\prime} \subset \Omega$. We consider the $\mathbf{C}$-algebra, with unit, of germs of analytic functions defined on open sets asymptotic to $\Gamma$, two such functions defining the same germ if they coincide on an open set asymptotic to $\Gamma$; and we say that an element $f$ in this algebra is asymptotic to an element $\hat{f}$ of $\mathbf{C}_{z}$ if for any integer $N \geq 1$ and any sector $\Gamma^{\prime} \subset \subset \Gamma$ we have, uniformly in $\Gamma^{\prime}$,

$$
f(z)=\sum_{r \leq N} f_{r} z^{r}+O\left(|z|^{N+1}\right) \quad\left(z \in \Gamma^{\prime}, z \longrightarrow 0\right) .
$$

The set of all $f$ for which such an $\hat{f}$ exists ( $\hat{f}$ is then unique) is denoted by $\mathcal{A}(\Gamma)$, and we denote the asymptotic condition by $f \sim \hat{f} \quad(\Gamma)$. It is easy to see that $\mathcal{A}(\Gamma)$ is a differential $\mathbf{C}$-algebra with unit and

$$
f \longmapsto \hat{f}
$$

is a homomorphism of differential algebras, namely, a homomorphism commuting with $\frac{d}{d z}$. The classical theorem of Borel-Ritt asserts that this is surjective ([18(a)], pp. 41-43). The elements of the kernel of this map are said to be flat on $\Gamma$. The classical (Cauchy) example of a flat function is $e^{-a / z^{N}}$ where $a=\alpha e^{i \varphi}$, flat on any sector in $\theta$ where $\cos (\varphi-N \theta)>0$.

We fix a sector $\Gamma$, an integer $m \geq 1$, and consider the system

$$
z^{m+1} \frac{d u_{i}}{d z}=\delta_{i} u_{i}+f_{i}\left(z: u_{1}, \ldots, u_{n}\right) \quad(1 \leq i \leq n)
$$

where

(i) the $\delta_{i}$ are nonzero complex numbers

(ii) the $f_{i}$ are polynomials in the $u$ 's with coefficients in the $\operatorname{ring} \mathcal{A}(\Gamma)$

(iii) the coefficients of the $f_{i}$ are asymptotically of order $\geq 0$; those of the terms of degree $\leq 1$ in the $u_{i}$ have asymptotic order $>0$.

The formalization of (1) is the system

$$
z^{m+1} \frac{d \hat{u}_{i}}{d z}=\delta_{i} \hat{u}_{i}+\hat{f}_{i}\left(z: \hat{u}_{1}, \ldots, \hat{u}_{n}\right) \quad(1 \leq i \leq n) .
$$

It makes sense to say that a solution $u$ to (1) is asymptotic to a solution $v$ to (2) on the sector $\Gamma$. The basic and beautiful theorem is then the following.

Theorem 1. Suppose that the vertex angle of $\Gamma$ is $\leq \pi / m$. If $v=\left(v_{i}\right)\left(v_{i} \in \mathbf{C}[[z]]\right)$ is a solution to the system (2) and the order of $v_{i}$ is $>0$ for all $i$, then we can find $u_{i} \in \mathcal{A}(\Gamma)$ such that

(i) $u=\left(u_{i}\right)$ is a solution to the system (1)

(ii) $u_{i} \sim v_{i} \quad(\Gamma)$.

For the proof refer to $[18(\mathrm{a})]$, pp. $65-76$ or $[8(\mathrm{~d})]$. There is a refinement with Gevrey asymptotics which plays a central role in the work of Ramis, Sibuya, and others; see [31] for this.

I shall now describe what is for us the main consequence of the above asymptotic existence theorem : asymptotic equivalence of meromorphic connections above a 
given formal equivalence. Let us consider two connection matrices $A_{1}, A_{2}$ from $\mathfrak{g l}\left(n, \mathbf{C}_{z, \text { cgt }}\right)$. Even though these may be equivalent under $G L\left(n, \mathbf{C}_{z}\right)$, one may not conclude from this that they are equivalent under $G L\left(n, \mathbf{C}_{z, \mathrm{cgt}}\right)$ because one cannot in general lift the relation

$$
\xi[A 1]=A_{2}, \quad\left(\xi \in G L\left(n, \mathbf{C}_{z}\right)\right)
$$

to a relation

$$
x\left[A_{1}\right]=A_{2}, \quad\left(x \in G L\left(n, \mathbf{C}_{z, \mathrm{cgt}}\right)\right) .
$$

But the asymptotic existence theorem of the preceding paragraph allows us to do this with $x$ belonging to $G L(n, \mathcal{A}(\Gamma))$. More precisely we have the following theorem.

Theorem 2. Let $\Gamma$ be any sector, and let $A_{i} \in \mathfrak{g l}(n, \mathcal{A}(\Gamma))(i=1,2)$ be such that their asymptotic expansions $\hat{A}_{i}$ are equivalent over $\mathbf{C}_{z}$; namely, for some $\xi \in$ $G L\left(n, \mathbf{C}_{z}\right)$ one has $\xi\left[\hat{A}_{1}\right]=\hat{A}_{2}$. Let $r_{1}$ be the common principal level of the $\hat{A}_{i}$. If the angle of $\Gamma$ is $\leq \pi /\left(\left|r_{1}\right|-1\right)$, we can find $x \in G L(n, \mathcal{A}(\Gamma))$ such that

(a) $x \sim \xi \quad(\Gamma)$

(b) $x\left[A_{1}\right]=A_{2}$ on $\Gamma$.

For the proof of this see [4(a)] [8(c)] [18(a)].

It must be remembered that the canonical levels of the connections $A_{i}$ need not be integers. But one overcomes this and works with unramified connections by going over to the plane of the variable $\zeta=z^{1 / b}$ for a sufficiently highly divisible integer $b \geq 1$. The proof, for which we may therefore assume that the $A_{i}$ are unramified, is by induction on $n$, and I only wish to comment on the key step which shows that it is essential to have the asymptotic existence theorem in the nonlinear context, although the theorem to be proved here is for linear equations.

The question is one of proving this when $\hat{A}_{2}=B$ is a canonical form with leading coefficient $D_{1}$ which is not a scalar. One then splits $\hat{A}_{2}$ as a direct sum along a spectrally disjoint splitting of $D_{1}$, and what one wants to do is to prove that this splitting can be lifted to a splitting of the connection $A_{1}$ by a gauge transformation that is asymptotic to the gauge transformation that one has at the formal level. We write $A$ for $A_{1}$ and assume that

$$
\hat{A}=D_{1} z^{r_{1}}+\hat{A}_{r_{1}+1} z^{r_{1}+1}+\ldots
$$

One can then show that we have an element

$$
\xi=\left(\begin{array}{cc}
1 & \tau \\
\tau & 1
\end{array}\right)
$$

where $\tau$ and $\tau^{\sim}$ are matrices with entries (in $\left.\mathbf{C}[[z]]\right) \equiv 0 \bmod z$ such that we have the formal equivalence

$$
\xi[\hat{A}]=\hat{B} .
$$

It is then a question of finding an $x \in G L(n, \mathcal{A}(\Gamma))$ of the form

$$
x=\left(\begin{array}{cc}
1 & t \\
t^{\sim} & 1
\end{array}\right)
$$


such that

$$
t \sim \tau, \quad t^{\sim} \sim \tau^{\sim}
$$

while $x[A]$ is in split form, i.e.,

$$
x[A]=\left(\begin{array}{ll}
* & 0 \\
0 & *
\end{array}\right) .
$$

Let us write $A_{i j}(1 \leq i, j \leq 2)$ for the block entries of $A$. If we compute $x[A]$ with unknown $t, t^{\sim}$ and write down the condition that the off-diagonal entries of $x[A]$ are 0 , we find nonlinear differential equations for $t$ and $t^{\sim}$. For $t$ (the treatment of $t^{\sim}$ is identical) the equation is of the Riccati type, namely

$$
\frac{d t}{d z}+t A_{22}-A_{11} t-t A_{21} t+A_{12}=0
$$

which has a quadratic nonlinearity in $t$. The existence of $t \sim \tau$ (and $t^{\sim} \sim \tau^{\sim}$ ) satisfying this equation (and its analogue for $t^{\sim}$ ) now follows from the asymptotic existence theorem.

The Stokes sheaf and the theorem of Malgrange-Sibuya. Let us fix $A_{0} \in$ $\mathfrak{g l}\left(n, \mathbf{C}_{z, \mathrm{cgt}}\right)$ and examine for any $A \in \mathfrak{g l}\left(n, \mathbf{C}_{z, \mathrm{cgt}}\right)$ the obstruction to the construction of an equivalence of $A$ with $A_{0}$ over $\mathbf{C}_{z, c g t}$, under the assumption (clearly necessary) that $A$ and $A_{0}$ are equivalent over $\mathbf{C}_{z}$. Let $\xi \in G L\left(n, \mathbf{C}_{z}\right)$ be such that $\xi\left[A_{1}\right]=A_{2}$. We can then construct a covering of $S^{1}$ by sectors $\Gamma$ with small angles and associated gauge transformations $x_{\Gamma} \in G L(n, \mathcal{A}(\Gamma))$ such that $x_{\Gamma} \sim \xi(\Gamma)$ and $x_{\Gamma}[A]=A_{0}$. But for any $\Gamma, x_{\Gamma}$ can be replaced by $u x_{\Gamma}$ where $u \in G L(n, \mathcal{A}(\Gamma)), u \sim 1, u\left[A_{0}\right]=A_{0}$. This suggests that we should introduce for each $\Gamma$ the set of all $u$ as above and try to solve the problem of making a coherent choice of these $u$ 's. The correct way to proceed, as Malgrange and Sibuya showed [28] [29], is to work with the cohomology of the sheaf of groups of flat sectorial automorphisms of the differential module $\left(\mathbf{C}_{z, \text { cgt }}^{n}, \nabla_{A_{0}}\right)$.

Let $S^{1}$ be the unit circle in the $z$-plane. For any $\theta \in S^{1}$ we consider matrix valued functions defined and analytic on regions of the form $\Gamma_{\delta}$ where $\delta>0$ and $\Gamma$ is a sector containing $\theta ; f \sim \xi(\theta)$, where $\xi$ is a matrix over $\mathbf{C}_{z}$, means that the asymptotic relation is true in some sector $\Gamma_{\delta}$ containing $\theta$.

Definition. Let $A_{0} \in \mathfrak{g l}\left(n, \mathbf{C}_{z, \text { cgt }}\right)$. The Stokes sheaf of $A_{0}, S t\left(A_{0}\right)$, is the sheaf of groups defined on $S^{1}$ whose stalk at any point $\theta \in S^{1}$ is the group of germs of analytic maps $u\left(\Gamma_{\delta} \longrightarrow G L(n, \mathbf{C})\right), \Gamma$ a sector containing $\theta$, such that

(i) $u \sim 1 \quad(\theta)$

(ii) $u\left[A_{0}\right]=A_{0}$.

We consider pairs $(A, \xi)$ where $A \in \mathfrak{g l}\left(n, \mathbf{C}_{z, \operatorname{cgt}}\right)$ and $\xi \in G L\left(n, \mathbf{C}_{z}\right)$ are such that $\xi[A]=A_{0}$; they are often called marked pairs. Two marked pairs $(A, \xi),\left(A^{\prime}, \xi^{\prime}\right)$ are equivalent if there is a $u \in G L\left(n, \mathbf{C}_{z, \text { cgt }}\right)$ such that $\xi^{\prime} \circ u=\xi$. Let $\mathfrak{M}\left(A_{0}\right)$ be the set of all equivalence classes of these pairs. Given a marked pair $(A, \xi)$, we can, in view of Theorem 1 , find a finite open covering $\left(U_{i}\right)$ of $S^{1}$ by $\operatorname{arcs} U_{i}$, a $\delta>0$, and holomorphic maps

$$
x_{i}: \Gamma\left(U_{i}\right)_{\delta} \longrightarrow G L(n, \mathbf{C})
$$


(for any arc $U$ of $S^{1}, \Gamma(U)$ is the sector of all points on the rays through the points of $U$ ) such that

$$
x_{i} \sim \xi \quad\left(\Gamma\left(U_{i}\right)\right), \quad x_{i}[A]=A_{0} .
$$

Then $\left(x_{i} x_{j}^{-1}\right)$ is a 1 -cocycle for $S t\left(A_{0}\right)$; the class of this cocycle does not depend on the covering or the choice of the $x_{i}$, and it also does not depend on the choice of the marked pair within the equivalence class in which it lies. We then have a well-defined map

$$
\Phi: \mathfrak{M}\left(A_{0}\right) \longrightarrow H^{1}\left(S^{1}, S t\left(A_{0}\right)\right) .
$$

The remarkable theorem of Malgrange and Sibuya [28] [29] [8(c)] is then the following.

Theorem 3 (Malgrange-Sibuya). The map $\Phi$ defined above is a bijection that maps the class of $\left(A_{0}, i d\right)$ on the trivial cohomology class.

The non-trivial part of the proof is the surjectivity of the map $\Phi$. To do this it is essential to show that any cocycle comes from some formal power series in the attempt to lift it to a convergent power series. That this is in fact true is another basic result of Malgrange and Sibuya (see [8(c)] for a detailed proof). Let $\mathcal{G}$ be the sheaf of groups on $S^{1}$ whose stalk at $t$ is the group of germs of holomorphic maps $g\left(\Gamma(W)_{\delta} \longrightarrow G L(n, \mathbf{C})\right)$ defined on sectors $\Gamma(W)_{\delta}$ containing $t \in S^{1}$, such that $g \sim 1(t)$. Thus $S t\left(A_{0}\right)$ is the subsheaf of $\mathcal{G}$ defined by the condition that $g\left[A_{0}\right]=A_{0}$. The same construction as above now leads to a well-defined map

$$
\Theta: G L(n, \mathbf{C}[[z]]) / G L(n, \mathbf{C}\{z\}) \longrightarrow H^{1}\left(S^{1}, \mathcal{G}\right) .
$$

Theorem 4 (Malgrange-Sibuya). The above map

$$
\Theta: G L(n, \mathbf{C}[[z]]) / G L(n, \mathbf{C}\{z\}) \longrightarrow H^{1}\left(S^{1}, \mathcal{G}\right)
$$

is a bijection of pointed spaces.

It is thus important to compute the Malgrange-Sibuya map in as many cases as possible. For the classical connections of Bessel and Whittaker this was done in $[8(\mathrm{c})]$ (see also [18(a)]). For confluent hypergeometric functions and their generalizations see the work of Duval and Mitschi [32], Mitschi [33], Ramis-Martinet [35(e)], and Loday-Richaud [30].

The Stokes sheaf as a sheaf of unipotent algebraic groups. If $A \in$ $\mathfrak{g l}\left(n, \mathbf{C}_{z, \text { cgt }}\right)$ is formally equivalent to $A_{0}$, the formal isomorphism can be lifted to an analytic isomorphism over $\mathcal{A}(\Gamma)$ for sectors $\Gamma$ with small vertex angles. It follows from this that the Stokes sheaves of $A_{0}$ and $A$ are locally isomorphic on $S^{1}$. Actually, even the global structure of $S t(A)$ can be determined from that of $\operatorname{St}\left(A_{0}\right)$ and the cohomology class that corresponds to $(A, \xi)$ in the Malgrange-Sibuya isomorphism, by the so-called process of twisting. More precisely, let $\gamma=\left(c_{i j}\right)$ be a cocycle associated to an open covering $\left(U_{i}\right)$ of $S^{1}$ by arcs that represent this cohomology class. Thus the $U_{i}$ are small enough to have isomorphisms $\gamma_{i}\left(A \sim A_{0}\right)$ on $U_{i}$ above $\xi$, and $c_{i j}=\gamma_{i} \gamma_{j}^{-1}$ on $U_{i} \cap U_{j}$. Then

$$
\left.\left.S t(A)\right|_{U_{i}} \simeq S t\left(A_{0}\right)\right|_{U_{i}},
$$


and $S t(A)$ is isomorphic to the sheaf obtained by gluing the sheaves $\mathcal{G}_{i}:=\left.S t\left(A_{0}\right)\right|_{U_{i}}$ along the $U_{i} \cap U_{j}$ by the isomorphisms

$$
\mathcal{G}_{j}(t) \simeq \mathcal{G}_{i}(t) \quad\left(t \in U_{i} \cap U_{j}\right)
$$

given by

$$
s \longmapsto c_{i j} s c_{i j}^{-1} \quad\left(s \in \mathcal{G}_{j}(t)\right) .
$$

This is the twisting process mentioned above, and we write

$$
S t(A)=S t\left(A_{0}\right)^{(\gamma)}
$$

One can also twist cohomology classes. Thus one has a twisting map

$$
\alpha \longmapsto \alpha^{(\gamma)}
$$

which gives a bijection

$$
H^{1}\left(S t\left(A_{0}\right)\right) \simeq H^{1}(S t(A)), \quad \gamma^{(\gamma)}=1,
$$

where we write 1 for the trivial class in $H^{1}(S t(A))$ (see [8(c)], pp. 110-116).

In view of this, for all local questions, and most global ones, it is enough to replace $S t(A)$ by $S t\left(A_{0}\right)$ for suitably chosen $A_{0}$. If the connections are unramified, we choose $A_{0}$ to be the canonical form; in the ramified case, we go over to a suitable covering plane and work with the unramified canonical form there. The sheaves $S t(A)$ on the $z$-plane and $S t\left(A^{\sim}\right)$ on the $\zeta$-plane where $A^{\sim}$ is the lifted connection from $A$ via the covering map $f: \zeta \longmapsto z$ are related sheaf-theoretically by

$$
\operatorname{St}\left(A^{\sim}\right)=f^{*}(S t(A)) .
$$

Let us illustrate this technique by determining the local structure of Stokes sheaves. We first assume that $A_{0}$ is an unramified canonical form

$$
B=D_{1} z^{r_{1}}+\cdots+D_{m} z^{r_{m}}+z^{-1} C .
$$

Let $\Sigma$ be the spectrum of the differential form $B d z$ so that we can represent $B d z$ and $C d z$ as block diagonal matrices with entries

$$
\sigma d z:=\sum_{j} c_{j}(\sigma) z^{r_{j}} d z \cdot 1, \quad C_{\sigma} d z .
$$

If $\psi$ is the block diagonal matrix with entries

$$
e^{-\int_{z}^{\infty} \sigma d z} \cdot 1
$$

an easy calculation shows that the map $g \longmapsto \psi^{-1} g \psi$ gives an isomorphism of $\operatorname{St}(B)$ with the sheaf $\mathcal{S}(B)$ of subgroups of $G L(n, \mathbf{C})$ whose stalks are defined by

$$
\mathcal{S}(B)(\theta)=\left\{h \in G L(n, \mathbf{C}) \mid \psi h \psi^{-1} \sim 1(\theta)\right\} .
$$


For $\sigma, \tau \in \Sigma$ let us write $\sigma<_{\theta} \tau$ to mean

$$
\exp \left(-\int_{z}^{\infty}(\sigma-\tau) d z\right) \sim 0(\theta) .
$$

Then

$$
h \in \mathcal{S}(B)(\theta) \Longleftrightarrow h_{\sigma \tau}= \begin{cases}1, & \text { if } \sigma=\tau \\ 0, & \text { unless } \sigma<_{\theta} \tau(\sigma \neq \tau) .\end{cases}
$$

The matrices $h$ are thus triangular with respect to $<_{\theta}$, so that the $\mathcal{S}(B)(\theta)$ are unipotent subgroups of $G L(n, \mathbf{C})$. For any open $\operatorname{arc} U, \mathcal{S}(U)$ is the unipotent subgroup of $G L(n, \mathbf{C})$ which is the intersection of all the $\mathcal{S}(\theta)$ for $\theta \in U$, and for open arcs $V \subset U$, the map $\mathcal{S}(U) \longrightarrow \mathcal{S}(V)$ is just the natural inclusion.

The asymptotic theorems discussed above now show that for any $A_{0}$ which is formally equivalent to $B, S t(A)$ is isomorphic to $S t(B)$ on any open arc of length $\leq \pi /\left(\left|r_{1}\right|-1\right), r_{1}$ being the principal level of $A$ (and $B$ ), and that any element of $H^{1}\left(S^{1}, S t(B)\right)$ trivializes on arcs of length $\pi /\left(\left|r_{1}\right|-1\right)$. These remarks, combined with the techniques of twisting and lifting to covering planes, then allow us to conclude that for any connection A, the Stokes sheaf $S t(A)$ is a sheaf of groups whose sections over open arcs have natural structures as unipotent linear algebraic groups, and that the restriction maps are morphisms of algebraic groups. We refer to the category of such sheaves of groups on $S^{1}$ as the category $\mathbf{A}$.

The equation $g\left[A_{0}\right]=A_{0}$ for determining the stalks is the same as the linear differential equation

$$
\frac{d g}{d z}+g A_{0}-A_{0} g=0
$$

which describes the differential equations for the horizontal sections of the connection ad $A_{0}$ (in the endomorphism bundle). We can now pass from the sheaf of unipotent groups to the sheaf of their Lie algebras to get a sheaf of (finite dimensional) nilpotent Lie algebras, determined by the same equations as above. We call this the infinitesimal Stokes sheaf and denote it by $s t\left(A_{0}\right)$. Its stalk at $\theta \in S^{1}$ is the Lie algebra of germs of holomorphic maps $a\left(\Gamma_{\delta} \longrightarrow \mathfrak{g l}(n, \mathbf{C})\right), \Gamma$ a sector containing $\theta$, such that

(i) $a \sim 0 \quad(\theta)$

(ii) $\frac{d a}{d z}+a A_{0}-A_{0} a=0$.

Unlike the nonabelian sheaf of groups defined earlier, this is a sheaf of complex vector spaces and so there is no problem of speaking about the cohomologies $H^{i}\left(S^{1}, s t\left(A_{0}\right)\right)$. The cohomology is 0 for $i \geq 2$ and $\operatorname{dim} H^{1}\left(S^{1}, s t\left(A_{0}\right)\right)$ is called the irregularity of the connection ad $A_{0}$; we write it as $\operatorname{Irr}\left(a d A_{0}\right)$.

Stokes lines. The Stokes sheaves are not locally constant. In fact, let us write, for any unramified canonical form $B$ and for any pair $\sigma, \tau$ of distinct elements of $\Sigma, S_{\sigma \tau}(B)$ for the finite set of rays passing through the points $\theta$ such that

$$
\gamma_{\sigma \tau}(\theta)=\operatorname{Re}\left(c_{a} \theta^{a+1} /(a+1)\right)=0,
$$

where

$$
\sigma-\tau=c_{a} z^{a}+\text { terms of higher order },
$$

and let

$$
S(B):=\bigcup_{\sigma \tau} S_{\sigma \tau}(B)
$$


Then, if $\theta$ does not lie on any of the rays from $S$, it is clear that $S t(B)\left(\theta^{\prime}\right)$ is unchanged as $\theta^{\prime}$ varies in a small neighborhood of $\theta$. However this is not the case if $\theta$ lies on one of the rays from $S_{\sigma \tau}(B)$ because $\gamma_{\sigma \tau}\left(\theta^{\prime}\right)$ is of opposite signs on the two sides of $\theta$ immediately adjacent to it. The elements of $S$ are called Stokes lines of the sheaf $S t(B)$ as well as the sheaf $S t(A)$ for any $A$ formally equivalent to $B$. If $A$ is ramified and becomes unramified by going over to the covering plane with covering $\operatorname{map} \zeta \longmapsto z=\zeta^{b}$, then the Stokes lines are defined by using the canonical forms in the $\zeta$-plane; the set of these lines is invariant under translations by the group of $b^{\text {th }}$ roots of unity, and so they define lines in the original $z$-plane, which are called the Stokes lines of $A$.

The fundamental fact about the Stokes lines, which follows from the local constancy of the sheaves on arcs that do not meet Stokes lines, is that they are the obstructions to analytically continuing the gauge equivalences above a given formal equivalence. Thus, let $A_{i}(i=1,2)$ be two connection matrices belonging to the same formal class over $\mathbf{C}_{z}$ as $A_{0}$ and let $\eta$ be an element of $G L\left(n, \mathbf{C}_{z}\right)$ such that $\eta\left[A_{1}\right]=A_{2}$. Suppose $W, W^{\prime}$ are open $\operatorname{arcs}$ of $S^{1}$ with $W^{\prime} \subset W$ and that $W \backslash W^{\prime}$ does not meet any Stokes line. If $y$ is an analytic map of $\Gamma\left(W^{\prime}\right)_{\delta}$ into $G L(n, \mathbf{C})$ such that $y \sim \eta\left(\Gamma\left(W^{\prime}\right)\right)$ and $y\left[A_{1}\right]=A_{2}$, then $y$ (which extends analytically to $\Gamma(W)_{\delta}$ and satisfies $y\left[A_{1}\right]=A_{2}$ there) remains asymptotic to $\eta$ on $\Gamma(W)$ also.

Elementary Stokes sheaves of level $r$ and the vanishing of their cohomology on arcs of length $\pi /(|r|-1)$. A connection $A$ in $\mathfrak{g l}\left(n, \mathbf{C}_{z, \operatorname{cgt}}\right)$ and the corresponding Stokes sheaf are called elementary if the canonical form of $A$ has only one level, say $r$; it is then said to be of level $r$. Note that we do not assume that $A$ is unramified. Let $b$ be an integer $\geq 1$ such that $A$ (and therefore its canonical form also) becomes unramified in the plane of $\zeta$ where $z=\zeta^{b}$. Let $A^{\sim}$ be the connection obtained by lifting $A$ via the covering map $f: \zeta \longmapsto z=\zeta^{b}$. The central fact about the elementary sheaves is the following (see [8(c)], p. 159), formulated by Deligne in his letters to Varadarajan $[9(\mathrm{~b})]$ in the more general context (see the next section) of sheaves of unipotent group schemes on $S^{1}$ :

Proposition 1. Let $A$ be an elementary connection of level $r$ whose lift to the plane of $\zeta=z^{1 / b}$ is unramified. Let $f$ be the covering map $\zeta \longmapsto z=\zeta^{b}$. Let $S^{1, b}$ be the unit circle in the $\zeta$-plane with the convention that its total length is $2 b \pi$. Then, for any arc $I \subset S^{1, b}$ of length $\pi /(|r|-1)$ whose endpoints are not on Stokes lines for $A$, we have

$$
H^{0}\left(I, f^{*}(S t(A))\right)=0, \quad H^{1}\left(I, f^{*}(S t(A))\right)=0 .
$$

This is the fundamental result that ultimately allows one to give a fairly complete description of the cohomology of the Stokes sheaf. I think it may already be in the work of Hukuhara, certainly for the part dealing with $H^{0}$, and partly and implicitly for the part dealing with $H^{1}$. The vanishing of $H^{0}$ is easy and is based on the simple fact that for any pair $\sigma, \tau$, there is exactly one Stokes line meeting the $\operatorname{arc} I$ and so for any section $g$ of the Stokes sheaf on $I$ one must have $g_{\sigma \tau}=0$; as $\sigma$ and $\tau$ are arbitrary, we have $g=1$. The vanishing of $H^{1}$ is more delicate. The trivialization of all cocycles on $I$ shows at once that the restriction map

$$
H^{1}\left(S^{1}, f^{*}(S t(A))\right) \longrightarrow H^{1}\left(I, f^{*}(S t(A))\right)
$$

is the 0 map; one then uses the vanishing of $H^{0}$ to extend this to the vanishing of $H^{1}\left(I, f^{*}(S t(A))\right)$ itself. 
Filtration of Stokes sheaves in terms of elementary ones. The second basic element of the structure theory of Stokes sheaves is that they possess a natural filtration of normal subsheaves whose successive subquotients are elementary. Let me now describe this (see [8(c), p. 154); it was formulated by Deligne [9(b)] for general sheaves of unipotent group schemes over $S^{1}$ (see the next section).

Let $A$ be a connection in $\mathfrak{g l}\left(n, \mathbf{C}_{z, \mathrm{cgt}}\right)$, not assumed to be unramified. For any $u \in S^{1}$ we write $\mathcal{D}(u)$ for the set of germs of holomorphic differential forms defined on sectors around $u$ of the form $\sum c_{a} z^{a} \cdot d z$ where the sums are finite and the $a$ are rational numbers $<-1$ (see [8(c)], p. 50). Then for any real number $t, S t(A)^{(t)}$ is the subsheaf of $\operatorname{St}(A)$ whose stalk at $u \in S^{1}$ is given by

$$
S t(A)^{(t)}(u)=\left\{g \in S t(A)(u) \mid(g-1) e^{-\int_{z}^{\infty} \omega} \sim 0(u) \text { if ord }(\omega)>t\right\} .
$$

The technique of twisting and lifting allows us to prove the following ([8(c), p. 154]). Let

$$
\left\{r_{1}<r_{2}<\cdots<r_{m}<-1\right\}
$$

be the canonical levels of $A$.

Proposition 2. The sheaves $S t(A)^{(t)}$ are normal subsheaves of $S t(A)$ decreasing with $t, 0$ for $t<r_{1}$, and $S t(A)$ for $t \geq r_{m}$. Moreover

$$
\operatorname{St}(A)^{(t)}=\operatorname{St}(A)^{\left(r_{k}\right)} \quad\left(r_{k} \leq t<r_{k+1}\right) .
$$

Write

$$
\operatorname{St}(A)^{(m-k)}=\operatorname{St}(A)^{(t)} \quad\left(t=r_{k}\right) .
$$

Then $\operatorname{St}(A)^{(i)} / \operatorname{St}(A)^{(j)}$ belongs to the category $\mathbf{A}$ for $j>i$,

$$
\operatorname{St}(A)=\operatorname{St}(A)^{(0)} \supset \operatorname{St}(A)^{(1)} \supset \cdots \supset \operatorname{St}(A)^{(m)}=0
$$

is a filtration by normal subsheaves, while $\operatorname{St}(A)^{(i)} / \operatorname{St}(A)^{(i+1)}$ is elementary with associated arc length $\pi /\left(\left|r_{m-i}\right|-1\right)$ for $i=0,1, \ldots, m-1$.

Summation methods, connections to the theory of resurgent functions, and Galois theory of linear differential equations. The methods of asymptotic analysis and cohomology that I have outlined thus far have their origin in the fact that the analytic solutions asymptotic to a formal solution are not uniquely determined. I think it was E. Borel who conceived of the idea of examining whether one can use summability methods to produce a distinguished analytic solution associated to its formal expansion. The advantage of such a procedure, when successful, is clear since it will allow one to pick out in a canonical manner a solution that is asymptotic to the formal one. Now, although the formal solutions do not converge, the coefficients of the formal solutions typically go to infinity in a manner related to the canonical levels so that one can try to use Gevrey asymptotics for the study of this question. Already in [13] Turrittin had raised the question of convergence of the formal solutions in this extended sense. This theme has been explored deeply by Ramis by himself and with his students and collaborators Martinet, Sibuya, Balser, Braaksma, Duval, Mitschi, Loday-Richaud, and others in recent years; and their work has shown beyond doubt that this theme is a profound one, with substantial 
applications to the structure and explicit determination of the Galois differential groups and the Stokes classes of meromorphic connections [7] [30] [31] [32] [33] . At the same time this method has linked the theory of meromorphic connections with the theory of resurgent functions developed by Ecalle [35]. One of the most interesting aspects of the work of Ramis is the discovery of a wild fundamental group at an irregular singularity whose representations characterize the connection up to meromorphic equivalence in the same way as the usual fundamental group does for a regular singularity [7]. I refer the reader to these references to get a better idea of these advances than what is conveyed by these very brief remarks. For an approach to Galois differential theory through Tannakian categories, see [9(c)].

\section{The SCheme Structure on $H^{1}\left(S^{1}, S t(A)\right)$. Moduli}

The classical examples of differential equations such as the Bessel, Whittaker, and other related equations with irregular singularities typically contain parameters whose variation does not change the formal structure of the connection. These are therefore examples of isoformal families of connections. Since $H^{1}\left(S^{1}, S t(A)\right)$ classifies the connections formally equivalent to $A$, it is natural to ask if this space is a moduli space for isoformal families; this of course requires that $H^{1}\left(S^{1}, S t(A)\right)$ be viewed as a complex analytic space in a natural manner. Since $H^{1}\left(S^{1}, S t(A)\right)$ is the cohomology of a sheaf of unipotent groups, and since sections of this sheaf are affine spaces, it may be expected that this might lead to the structure of a variety on the cohomology. There are of course complications since we deal with nonabelian cohomology.

Already in his letters to Malgrange, Deligne had noted that $H^{1}\left(S^{1}, S t\right)$ was a smooth variety. The classical treatment of the question of a natural parametrization is to be found in the works of Balser, Jurkat, and Lutz [16(a)(b)(c)]. These authors showed (in our language, see below) that for certain good coverings $\mathfrak{A}$ of the universal cover of $S^{1}$ one has $H^{1}\left(S^{1}, S t\left(A_{0}\right)\right)=H^{1}\left(\mathfrak{A}, S t\left(A_{0}\right)\right)$ is an affine space whose dimension is the irregularity $\operatorname{Irr}\left(a d A_{0}\right)$. Similar good coverings also play a vital role in the explicit calculations treated in the work of Loday-Richaud [30]. In this section I summarize the cohomological approach to the structure of $H^{1}\left(S^{1}, S t(A)\right)$ from the more canonical and categorical point of view that was developed in [8(c)], following $[8(\mathrm{f})(\mathrm{g})]$, but influenced deeply by the ideas of Deligne in [9(b)].

The method of studying the variety structure of $H^{1}\left(S^{1}, S t\right)$ is quite simple basically. For each covering $\mathfrak{A}$ of $S^{1}$ we have the group of coboundaries $C(\mathfrak{A})$ which acts on the set $Z(\mathfrak{A})$ of cocycles; the equations defining the cocycles make the space $Z(\mathfrak{A})$ an affine variety in a natural manner, the structure deriving from the structure of the unipotent groups that is part of the data of the Stokes sheaf. The coboundary action is morphic. The cohomology associated to the covering is thus the orbit space $Z(\mathfrak{A}) / C(\mathfrak{A})$. The problem is one of showing that this orbit space with its quotient structure is a nice variety for a cofinal family of coverings, and that the variety structure does not depend on the cover. It will turn out that the coboundary action is free for certain good coverings, at least set theoretically, and also that the refinement maps are bijective. But to prove these facts morphically is another matter and requires involved arguments from classical algebraic geometry. Moreover, this cannot be done directly for the connection itself, and one has to use a filtration of the structure in terms of connections which are elementary in the sense described earlier, i.e., having just one canonical level. This was the proce- 
dure followed in $[8(\mathrm{f})(\mathrm{g})]$, where only unramified connections were treated. Finally, ramification produces its own complications; one has to go up to covering planes, and then come down.

In his letters [9(b)] to Varadarajan, Deligne pointed out that if one operates scheme-theoretically, then a considerable amount of technical difficulty can be eliminated. Roughly speaking, the idea is that all natural set theoretic calculations which are made over $\mathbf{C}$ remain true for algebras $R$ over $\mathbf{C}$ and give morphic properties-for instance, the bijectivities produced by the refinement maps will be automatically morphisms if verified for all $\mathbf{C}$-algebras $R$. To operate at the scheme level, one has to formulate the Stokes data scheme-theoretically. This is done by observing that a unipotent group over $\mathbf{C}$ gives rise to a reduced unipotent group scheme over $\mathbf{C}$ and using this idea to pass from the sheaf of unipotent groups to a sheaf of unipotent group schemes. The cohomology $H^{1}\left(S^{1}, S t\right)$ now becomes a functor from $\mathbf{C}$-algebras to sets, and the problem is to show that this is representable by affine space. I shall now give a brief description of the highlights of this method. For full details one should refer to [8(c)].

We recall the category $\mathbf{A}$ of sheaves $G$ of groups on $S^{1}$ which are unipotent algebraic over all open arcs; $S t(A)$ has this property for any connection $A$. We replace for any open arc $U$ the group $G(U)$ by the corresponding reduced group scheme $\mathcal{G}(U)$. The unipotency then allows one to establish the following fundamental fact: for any $\mathbf{C}$-algebra $R$, the assignment

$$
U \longmapsto \mathcal{G}(U)(R)
$$

is already the restriction to the arcs of a sheaf of groups on $S^{1}$, denoted by $\mathcal{G}(R)$, and the assignment

$$
R \longmapsto \mathcal{G}(R)
$$

is a covariant functor from the category of $\mathbf{C}$-algebras to the category of sheaves of groups on $S^{1}$. We call such a functor a sheaf of unipotent group schemes on $S^{1}$. Thus, for any connection $A$ we may regard $S t(A)$ as a sheaf of unipotent group schemes on $S^{1}$, again denoted by $S t(A)$. The unipotency is critical in establishing this fact.

Given a sheaf $\mathcal{G}$ of unipotent group schemes on $S^{1}$ it is now clear that we can form the functors

$$
H^{i}(U, \mathcal{G}): R \longmapsto H^{i}(U, \mathcal{G}(R))
$$

from the category of $\mathbf{C}$-algebras to the category of sets, $U$ being any open arc, including the possibility $U=S^{1}$ also. The question is to examine to what extent these functors are representable. I shall now formulate a context in which one can show that the functor

$$
H^{1}\left(S^{1}, \mathcal{G}\right): R \longmapsto H^{1}\left(S^{1}, \mathcal{G}(R)\right)
$$

is representable by affine space; the conditions will always be satisfied by the Stokes sheaf, and in fact, they are formulated by distilling the essence of the arguments in $[8(\mathrm{f})(\mathrm{g})]$, as was done in $[9(\mathrm{~b})]$.

We shall work with the covering circles $S^{1, b}$, covering maps $f_{b}: \zeta \longmapsto z=\zeta^{b}$, and use the convention that the total length of $S^{1, b}$ is $2 b \pi$. A sheaf $\mathcal{U}$ of unipotent group schemes on $S^{1}$ is called elementary if the following is satisfied: there is a 
number $a(\mathcal{U})>0$ such that $0<a(\mathcal{U})<2 b \pi$ and a finite set $\Phi \subset S^{1, b}$ such that for all arcs (always open) $I$ in $S^{1, b}$ of length $a(\mathcal{U})$ whose endpoints are not in $\Phi$, we have

$$
H^{0}\left(I, f_{b}^{*}(\mathcal{U}(R))=0, \quad H^{1}\left(I, f_{b}^{*}(\mathcal{U}(R))=0\right.\right.
$$

for all $\mathbf{C}$-algebras $R$. The basic theorem is then the following ([8(c)], II, Theorem 2.4.1, p. 134).

Theorem 1. Let $\mathcal{U}$ be a sheaf of unipotent group schemes on $S^{1}$ with a filtration

$$
\mathcal{U}=\mathcal{U}^{(0)} \supset \mathcal{U}^{(1)} \supset \cdots \supset \ldots \quad\left(\mathcal{U}^{(i)}=0 \text { for } i>>0\right)
$$

such that $\mathcal{V}^{(i)}:=\mathcal{U}^{(i)} / \mathcal{U}^{(i+1)}$ and $\mathcal{U} / \mathcal{U}^{(i)}$ are from the category $\mathbf{A}$ for all $i$. Suppose that the $\mathcal{V}^{(i)}$ are elementary with arc length $a\left(\mathcal{V}^{(i)}\right)=: a_{i}$ for all $i$ and that

$$
a_{0} \geq a_{1} \geq a_{2} \geq \ldots
$$

Then

(i) for any $\mathbf{C}$-algebra $R$ and any 1-cocycle $\alpha$ from $\mathcal{U}(R)$,

$$
H^{0}\left(S^{1}, \mathcal{U}^{(\alpha)}\right)=0
$$

where the superscript refers to the twisting by $\alpha$

(ii) for any 1-cocycle $\lambda$ from $\mathcal{U}(\mathbf{C})$ the twisted sheaves of group schemes $\mathcal{U}^{(\lambda)}$ are from $\mathbf{A}$, have the same properties as $\mathcal{U}$ with respect to the filtration $\mathcal{U}^{(\lambda)}$, the associated arc lengths remaining the same

(iii) the functor

$$
R \longmapsto H^{1}\left(S^{1}, \mathcal{U}(R)\right)
$$

is representable by affine space.

The proof is of course in two parts: the first part is to prove it for elementary sheaves, and then use the exact sequences

$$
0 \longrightarrow \mathcal{V}^{(i)}(R) \longrightarrow \mathcal{U} / \mathcal{U}^{(i+1)}(R) \longrightarrow \mathcal{U} / \mathcal{U}^{(i)}(R) \longrightarrow 0
$$

to prove it for $\mathcal{U} / \mathcal{U}^{(i)}$ by induction on $i$. If $\mathcal{U}$ is elementary with arc length $a$, then there are two cases: $a \leq 2 \pi$ and $a>2 \pi$. In the first case the $\operatorname{arcs} I$ with vanishing cohomologies can be taken to be in $S^{1}$ itself, and one works with a covering of $S^{1}$ by arcs of length $a$, with the covering having either no triple intersections $(\pi<a \leq 2 \pi)$ or all distinct triple intersections empty $(0<a \leq \pi)$. The vanishing of $H^{0}$ for such arcs now tells us that the coboundary group is trivial and the cocycle condition is vacuous, while the vanishing of $H^{1}$ tells us that the cohomology is already determined by this cover. The cohomology is thus the product of the groups of sections of arcs (or disjoint finite unions of arcs) and so is representable by affine space since the group of points of a unipotent group scheme is representable by affine space. The case $a>2 \pi$ is more subtle; in this case, if $J$ is an arc in the covering circle (that is part of the definition of elementary) of length $a-2 \pi$ with ends not on the finite set $\Phi$, one shows that there is a functorial isomorphism

$$
H^{1}\left(S^{1}, \mathcal{U}(R)\right) \simeq H^{0}\left(J, f^{*}(\mathcal{U}(R))\right.
$$


(see [8(c)], II, Proposition 1.5.1, p. 121) which gives the representability by affine space for $H^{1}$.

For the inductive step one uses the following principle ([8(c)], II, Lemma 2.5.3, p 139): if $F$ and $G$ are two functors from $\mathbf{C}$-algebras to sets with a natural map $u(F \longrightarrow G)$, then for the representability of $F$ by affine space it is sufficient that (i) $G$ is representable by affine space and (ii) for any $\mathbf{C}$-algebra $R$ and any $g \in G(R)$, the fibre above $g$, namely the functor $S \longmapsto u(S)^{-1}(g)$ from $R$-algebras $S$ to sets, is representable by affine space over $R$. First one shows that all the sheaves involved and their twists have vanishing $H^{0}$ :

$$
H^{0}\left(S^{1}, \mathcal{U} / \mathcal{U}^{(i)}(R)^{(\alpha)}\right)=0
$$

for any $\mathbf{C}$-algebra $R$, and any 1-cocycle $\alpha$ for $\mathcal{U} / \mathcal{U}^{(i)}(R)$. We then have the exact sequence coming from $(E)$, namely,

$$
0 \longrightarrow H^{1}\left(S^{1}, \mathcal{V}^{(i)}(R)\right) \longrightarrow H^{1}\left(S^{1}, \mathcal{U} / \mathcal{U}^{(i+1)}(R)\right) \longrightarrow H^{1}\left(S^{1}, \mathcal{U} / \mathcal{U}^{(i)}(R)\right) \longrightarrow 0 .
$$

The principle referred to above now establishes the representability by induction on $i$.

There is one technical point here worth noting. The principle mentioned above requires verifying the representability over $R$ of the fiber in $H^{1}\left(S^{1}, \mathcal{U} / \mathcal{U}^{(i+1)}(S)\right)$ above any point of $H^{1}\left(S^{1}, \mathcal{U} / \mathcal{U}^{(i)}(R)\right)$. But exact sequences involving pointed spaces have implications only about fibers over the distinguished points. It is here that the twists come into the picture. Twisting allows one to transfer a given cohomology class to the trivial class of a twisted sheaf. Indeed, if we have the exact sequence of sheaves of groups

$$
0 \longrightarrow \mathcal{G}^{(b)} \longrightarrow \mathcal{G}^{(b)} \longrightarrow \mathcal{G}^{\prime \prime(a)} \longrightarrow 0
$$

where the superfixes $a$ and $b$ refer to compatible twistings, then we have the exact sequence below that makes precise the moving of the fibers that was mentioned above (the down maps are the twistings by the cocycles $b$ and $a$ ):

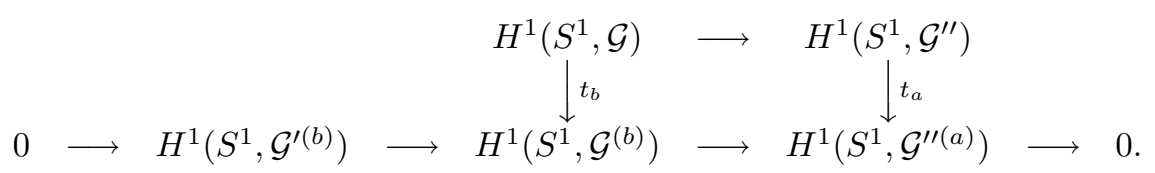

We now apply this idea to the twisted exact sequence

$$
0 \longrightarrow \mathcal{V}^{(i)}(R)^{(\beta)} \longrightarrow \mathcal{U} / \mathcal{U}^{(i+1)}(R)^{(\beta)} \longrightarrow \mathcal{U} / \mathcal{U}^{(i)}(R)^{(\alpha)} \longrightarrow 0 .
$$

The vanishing of the $H^{0}$ of the twisted sheaves now comes into play and gives us an exact sequence of $H^{1}$ spaces, and the fiber above the distinguished point is the object to be treated.

To apply this theorem to the Stokes sheaf, we must verify that being elementary in the sense of group schemes, where the vanishing of the cohomologies over appropriate arcs is required for all $\mathbf{C}$-algebras, is guaranteed for the elementary Stokes sheaves of $\S 6$. This is a technical point and involves invoking the Zariski Main Theorem in the context of affine spaces ([8(c)], II, Proposition 2.4.2, p. 135). 
It is an easy result in the theory of sheaves of groups that if $\mathcal{G}$ is a sheaf of groups on a topological space $X$ and $\alpha$ is any 1-cocycle such that one has

$$
H^{0}\left(X, \mathcal{G}^{(\alpha)}\right)=0,
$$

then the action of the coboundary group (for the covering defining $\alpha$ ) on the orbit of $\alpha$ is free ([8(c)], II, Proposition 1.3.1, p. 115). The vanishing of the $H^{0}$ of the twisted sheaves in the above theorem now leads to the following result which describes the formation of the cohomology from the cocycle spaces in a very transparent manner. Let $\mathcal{U}$ be a sheaf of unipotent group schemes satisfying the conditions of Theorem 1. A covering $\mathfrak{A}$ of $S^{1}$ is called good if

$$
H^{1}\left(S^{1}, \mathcal{U}(R)\right)=H^{1}(\mathfrak{A}, \mathcal{U}(R))
$$

for all C-algebras $R$. It can be shown ([8(c)], II, Proposition 2.7.1, p. 147) that any finite cover by open arcs of length $a(\mathcal{U}):=\min _{i} a_{i}(\mathcal{U})$ with ends not in the appropriate finite set (Stokes lines!) is a good cover. The vanishing of the $H^{0}$ of the twisted sheaves now implies that for any $\mathbf{C}$-algebra $R$, the coboundary group $C(\mathfrak{A}: R)$ acts freely on the cocycle space $Z(\mathfrak{A}: R)$, while the representability theorem implies that the cocycle space functor

$$
R \longmapsto Z(\mathfrak{A}: R)
$$

is representable by affine space over $\mathbf{C}$. The coboundary groups are trivially representable by affine spaces. We then have the following theorem ([8(c)], II, Theorem 2.7.6, p. 149).

Theorem 2. Let $\mathfrak{A}$ be a good covering. Then the action of $C(\mathfrak{A}: \mathbf{C})$ on $Z(\mathfrak{A}: \mathbf{C})$ is free in the algebraic geometric sense, and the map

$$
Z(\mathfrak{A}: \mathbf{C}) \longrightarrow H^{1}\left(S^{1}, \mathcal{U}(\mathbf{C})\right)=C(\mathfrak{A}: \mathcal{U}(\mathbf{C}) \backslash Z(\mathfrak{A}: \mathcal{U}(\mathbf{C}))
$$

is the quotient map.

The reader who wishes to get a more detailed view of how the coverings, ramifications, coboundary actions and so on fit together, and how they are related to the theory of Galois differential groups and the summability processes should refer to the account of these issues given in [30].

\section{REFERENCES}

1. B. Riemann, Beiträge zur Theorie der durch die Gauss'sche Reihe $F(\alpha, \beta, \gamma, x)$ darstellbaren Funktionen, Abh. Kon. Ges. d. Wiss. zu Göttingen VII Math. Classe, A-22 (1857); Collected Papers (Raghavan Narasimhan, ed.), Springer-Verlag, Berlin, 1990, pp. 99-119.

2. J. Gray, (a) Fuchs and the theory of differential equations, Bull. Amer. Math. Soc. 10, No. 1 (1984), 1-26 MR 85h:0102b; (b) Linear Differential Equations and Group Theory from Riemann to Poincaré, Birkhäuser, Boston, Basel, 1986. MR 85h:0102a

3. V.I. Arnold and Yu.S. Il'yashenko, Dynamical Systems I, vol. 1 of Encyclopaedia of Mathematical Sciences (D.V. Anosov and V.I. Arnold, eds.), Springer, New York, 1988.

4. (a) V.S. Varadarajan, Meromorphic Differential Equations, Expositiones Mathematicae 9, No.2 (1991), 97-188 MR 91i:32024; (b) Some remarks on meromorphic differential equations with simple singularities, Bull. Calcutta Math. Soc. Diamond Jubilee Volume (1983), 49-61 MR 87k:35051; (c) A. Treibich Kohn, Un resultat de Plemelj, "Mathematique et Physique", Sem. Ecole. Norm. Sup. (eds. L. Boutet de Monvel, A. Douady, and J. L. Verdier), Birkhäuser, 1983. 
5. (a) D. Bertrand, Travaux récent sur les points singuliers des équations différentielle linéaires, Springer Lecture Notes in Mathematics-Sém. Bourbaki, 1978/79, Exposés 525-542, 770(1980); (b) Groupes algébriques et équations différentielles linéaires, Sém. Bourbaki, 1991-1992, Exposé $\mathrm{n}^{\circ} 750$, (c) A. Beauville, Monodromie des systèmes différentiels linéaires à pôles simples sur la sphère Riemann, Sém. Bourbaki, 1992-1993, Exposé $\mathrm{n}^{\circ} 765$.

6. (a) D. V. Anosov and A. Bolibruch, The Riemann-Hilbert problem, The Steklov Mathematical Institute, Moscow, Vieweg, 1994 MR 95d:32024;(b) V. Kostov, Fuchsian linear systems on $\mathbf{C P}^{1}$ and Riemann-Hilbert's problem, Prépublication Université de Nice (1991); Fuchsian linear systems on $\mathbf{C P}^{1}$ and the Riemann-Hilbert problem, C. R. Acad. Sci. Paris. Ser. I, t. 315 (1992), 143-148;(c) K. Iwasaki, H. Kimura, S. Shimomura, and M. Yoshida, From Gauss to Painlevé : a modern theory of special functions, Vieweg, 1991 MR 94a:34007;(d) K. Iwasaki, Moduli and deformation for Fuchsian projective connections on a Riemann surface, Jour. of the Fac. of Sci., Univ. of Tokyo, Sec. IA 38 (1991), 431-531; (e)Fuchsian moduli on a Riemann surface-its Poisson structure and Poincaré-Lefschetz duality, Pacific Jour. Math. 135 (1992), 319-340 MR 93i:32028;(f) M. F. Singer, An outline of differential Galois theory, Computer algebra and differential equations (E. Tournier, ed.), Academic Press, 1991, pp. 1-57, MR 91a:12011; (g) Moduli of linear differential equations on the Riemann sphere with fixed Galois groups, Pacific. Jour. of Math. 160, 343-395.

7. J. P. Ramis, (a) Phénomène de Stokes et filtration Gevrey sur le groupe de Picard-Vessiot, C. R. Acad. Sci. Paris, Ser. I, t. 301 (1985), 165-167 MR 86k:12012; (b) Phénomène de Stokes et resommation, C. R. Acad. Sci. Paris, Ser. I, t. 301 (1985), 99-102 MR 86k:12011; (c) Filtration Gevrey sur le groupe de Picard-Vessiot d'une équation différentielle irréguliere, Preprint IMPA, Rio de Janeiro 45 (1985); (d) Irregular connections, savage $\pi_{1}$, and confluence, Conference in Katata, Taniguchi Foundation, Preprint, 1988; (e) Les series k-summables et leurs applications, Analysis, microlocal analysis and relativistic quantum field theory, Springer Lecture Notes in Physics 126 (1980), 178-197; (f) Confluence and resurgence, Jour. Fac. Sci. Univ. Tokyo, Sec. IA, 36 (1989), 703-716; (g) Divergent series and holomorphic dynamical systems, Preprint, 1993.

8. D.G. Babbitt and V.S. Varadarajan, (a) Formal reduction of meromorphic differential equations: a group theoretic view, Pacific J. Math. 108 (1983), 1-80 MR 86b:34010; (b) Deformations of nilpotent matrices over rings and reduction of analytic families of meromorphic differential equations, Mem. Amer. Math. Soc. 55 (1985), no. 325, MR 87i:12014; (c) Local Moduli for meromorphic differential equations, Astérisque 169-170 (1989), 1-217 MR 91e:32017; (d) Some remarks on the asymptotic existence theorem for meromorphic differential equations, Jour. of the Fac. of Sci, Univ. of Tokyo, Sec IA, 36 (1989), 247-262 MR 91b:34006; (e) Meromorphic connections with irregular singularities : some problems, Katata conference and workshop, Taniguchi Foundation (1987), Preprint; (f) Local moduli for meromorphic differential equations. I. The Stokes sheaf and its cohomology, UCLA, preprint (1985); (g) Local isoformal deformation theory for meromorphic differential equations near an irregular singularity (M. Hazewinkel and M. Gerstenhaber, eds.), Deformation theory of algebras and structures and applications, NATO ASI Series C. Mathematical and Physical Sciences, Vol. 247, Kluwer Academic Publishers, 1988, pp. 583-700; (h) D. G. Babbitt, Groupes algébriques et réduction formelle de systèmes différentielles linéaires, Publ. Math. Univ. Pierre et Marie Curie 84 (1986-87), II.1-II.16.

9. (a) P. Deligne, Equations Différentielles à points singuliers régulier, Springer Lecture Notes in Mathematics 163 (1970), MR 54:5232; (b) Letters to Malgrange, December 12, 1976; 22 August, 1977; April, 1978; Letters to Varadarajan, 4 January, 1986, 2 February, 1986; Letters to Ramis, January 1, 1986; February 25, 1986; February 28, 1986; (c) Catégories tannakiennes, Grothendieck Festschrift (P. Cartier et al., eds.), Birkhäuser, 1991, pp. 111195.

10. (a) Yu. Manin, Moduli Fuchsiani, Ann. Sc. Norm. Sup. Pisa 19 (1965), 113-126 MR 31:4815; (b) N. Katz, Nilpotent connections and the monodromy theorem: application of a result of Turrittin, Publ. Math. IHES 39 (1970), 207-238. MR 45:271

11. A. Borel et al., Algebraic D-Modules, Academic Press Inc., Boston/New York, 1987. MR 89g:32014

12. M. Hukuhara, (a) Sur les points singuliers des équations différentielles linéaires, II, Jour. Fac. Sci. Hokkaido Univ. 5 (1937), 123-166.(b) Sur les points singuliers des équations différentielles linéaires, III, Jour. Fac. Sci. Kyushu Univ. 2 (1942), 125-137. MR 9:92i 
13. H. Turrittin, Convergent solutions of ordinary differential equations in the neighborhood of an irregular singular point, Acta Math. 93 (1955), 27-66. MR 16:925a

14. A.H.M. Levelt, Jordan decomposition for a class of singular differential operators, Arkiv för matematik 13(1) (1975), 1-27. MR 58:17962

15. (a) E. Fabry, Sur les intégrales des équations différentielles linéaires à coefficients rationnels, Thése, Paris, 1885;(b) H. Poincaré, Sur les intégrales des équations linéaires, Acta Math. 8 (1986), 295-344.

16. (a) W. Balser, Zum Einzigkeitssatz in der Invariantentheorie meromorpher Differentialgleichungen, Jour. für die reine und angewandte Mathematik 318 (1980), 51-82 MR 82d:34006; (b) W. Balser, W. Jurkat, and D. Lutz, A general theory of invariants for meromorphic differential equations; Part II, Proper invariants, Funkcialaj Ekvacioj 22 (1979), 257-283 MR 83m:34003b;(c) W. Jurkat, Meromorphe Differentialgleichungen, Springer Lecture Notes in Math 637 (1978). MR 82a:34004

17. G. Appleby, Thesis, UCLA, 1993.

18. W. Wasow, (a) Asymptotic expansions for ordinary differential equations, Dover, 1987; (b) Linear Turning Point Theory, Springer-Verlag, New York, 1985. MR 86f:34114

19. W. Bruns, E. G. Evans, Jr. and P.A. Griffith, Syzygies, Ideals of Height Two, and Vector Bundles, J. of Algebra 67 (1980), 143-162. MR 82e:13009

20. F. Beukers and G. Heckman, Monodromy for the hypergeometric function ${ }_{n} F_{n-1}$, Inventiones Math. 95 (1989), 325-354. MR 90f: 11034

21. (a) P. Candelas, X. C. de la Ossa, P. S. Green, and L. Parkes, A pair of Calabi-Yau manifolds as an exactly soluble superconformal theory, Nucl. Phys. B 359 (1991), 21-74 MR 93b:32029; (b) A. Cadavid and S. Ferrara, Picard-Fuchs equations and the moduli space of super conformal field theories, Phys. Lett. B 267 (1991), 193-199 MR 92i:32022;(c) D. R. Morrison, Picard-Fuchs equations and mirror maps for hypersurfaces, Essays on Mirror Manifolds (S. T. Yau, ed.), International Press, 1992, pp. 241-264. MR 94b:32035

22. Harish-Chandra, (a) Some results on differential equations and their applications, Proc. Nat. Acad. Sci. USA 45 (1959), 1763-1764; Collected Papers, Volume III, Springer Verlag, 1984, pp. 7-56. MR 85e:01061c

23. I. M. Gel'fand, Collected papers, Volume III, Part V, Springer Verlag, 1989. MR 90d:01091

24. M. Yoshida, Fuchsian differential equations, Vieweg, 1987. MR 90f:32025

25. (a) G. J. Heckman and E. M. Opdam, Root systems and hypergeometric functions, I. Comp. Math. 64 (1988), 329-352; III. Comp. Math. 67 (1988), 21-49; IV. Comp. Math. 67 (1988), 191-209 MR 89b:58192a;(b) G. J. Heckman, Root systems and hypergeometric functions, II. Comp. Math. 64 (1987), 353-373. MR 89b:58192b

26. (a) R. Sommeling, Characteristic classes for irregular singularities, Thesis, University of Nijmegen, 1993;(b) A. H. M. Levelt, Stabilizing differential operators: a method for computing invariants at irregular singularities, Differential equations and computer algebra (M. F. Singer, ed.), pp. 181-228, Academic Press, 1991. MR 92h:34013;(c) D. G. Babbitt and V. S. Varadarajan, Formal differential equations containing a parameter, Differential equations and computer algebra (M. F. Singer, ed.), Academic Press, 1991, pp. 77-111. MR 92i:32023

27. (a) H. Majima, Asymptotic analysis for integrable connections with irregular singular points, Springer Lecture Notes in Mathematics, 1984, p. 1075, MR 86e:58004;(b) A. H. M. Levelt and A. van den Essen, Irregular singularities in several variables, Mem. Amer. Math. Soc. 40 (1982). MR 84e:13031

28. B. Malgrange, Remarques sur les équations différentielles à points singulier irréguliers, Equations Différentielles et Systèmes de Pfaff dans le champ complexe, Springer Lecture Notes in Mathematics 712 (1979). MR 80k:14019

29. Y. Sibuya, Stokes phenomena, Bull. Amer. Math. Soc. 83 (1977), 1075-1077. MR 56:720

30. M. Loday-Richaud, Prépublications, Université de Paris-Sud Mathematiques, Orsay, 1992.

31. J. P. Ramis and Y. Sibuya, Hukuhara's domains and fundamental existence and uniqueness theorems for asymptotic solutions of Gevrey type, Asymptotic Analysis 2 (1989), 39-94. MR 90k:58209

32. A. Duval and C. Mitschi, Matrices de Stokes et groupe de Galois des équations hypergeometriques confluents generalisées, Pacific J. of Math 138 (1989), 25-56. MR 91c:14015

33. C. Mitschi, Groupe de Galois différentiel des équations hypergéométriques confluentes géneralisés, C. R. Acad. Sci. Paris, t. 309, Ser. I (1989), 217-220. MR 91a:12010 
34. (a) W. Balser, B. L. J. Braaksma, J. P. Ramis, and Y. Sibuya, Asymptotic analysis, Multisummability of formal power series solutions of linear ordinary differential equations 5 (1991), 27-45 MR 93f:34011;(b) B. L. J. Braaksma, Multisummability and Stokes multipliers for linear meromorphic differential equations, J. Diff. Equations 92 (1991), 45-75. MR 93c:34010

35. (a) J. Ecalle, Les fonctions résurgents, Publ. Math. Orsay, I, II. (1981); III. (1985) MR 87k:32009;(b) W. Balser, From divergent power series to analytic functions: theory and applications of multisummable power series, Lecture Notes in Mathematics, Springer, vol. 1582, 1994;(c) Y. Sibuya, Gevrey asymptotics and Stokes multipliers, Differential equations and computer algebra (M. F. Singer, ed.), Academic Press, 1991, pp. 131-147;(d) B. Candelpergher, J. C. Nosmas, and F. Pham, Approche de la résurgence, Hermann, Paris, 1993 MR 95e:34005;(e) J. P. Ramis and J. Martinet, Differential equations and computer algebra (E. Tournier, ed.), Academic Press, 1990, pp. 117-214. MR 91d:12014; (f) Elementary acceleration and multisummability, Publ. Univ. Louis Pasteur, 1990.

Department of Mathematics, University of California at Los Angeles, Los Angeles, CA 90095-1555

E-mail address: vsv@math.ucla.edu 